\title{
Functional norms for Young towers
}

\author{
MARK F. DEMERS \\ Department of Mathematics, Fairfield University, Fairfield CT 06824, USA \\ (e-mail: mdemers@fairfield.edu)
}

(Received 15 January 2009 and accepted in revised form 22 May 2009)

\begin{abstract}
We introduce functional norms for hyperbolic Young towers which allow us to directly study the transfer operator on the full tower. By eliminating the need for secondary expanding towers commonly employed in this context, this approach simplifies and expands the analysis of this class of Markov extensions and the underlying systems for which they are constructed. As an example, we prove large-deviation estimates with a uniform rate function for a large class of non-invariant measures and show how to translate these to the underlying system.
\end{abstract}

\section{Introduction}

Young towers were introduced in [Y1] as a unified framework in which to view the statistical properties of both uniformly and non-uniformly hyperbolic dynamical systems. Briefly, given a dynamical system $f: M \circlearrowleft$, a Young tower is a type of Markov extension $F: \Delta \circlearrowleft$ with the following representation. Given a reference measure $\mu$, one chooses a reference set $\Lambda \subset M$ of positive measure with a hyperbolic product structure and constructs a return time function $R: \Lambda \rightarrow \mathbb{Z}^{+}$with certain (Markov) properties. The Young tower is an extension of $\bigcup_{\ell \geq 0} f^{\ell} \Lambda$ where the $\ell$ th level of the tower corresponds to those $x \in f^{\ell} \Lambda$ for which $R(x)>\ell$. In essence, the Young tower represents $\Lambda$ as a horseshoe with countably many branches and variable return times. The rate of decay in the measure of the levels of the tower, $\mu(R(x)>\ell)$, gives information about the statistical properties of $f$; for example, it reflects the exponential or polynomial decay of correlations (see $[\mathbf{C Y}]$ for a survey).

Young towers have been constructed for many systems: billiards with convex scatterers, including those subject to external forces [C3, C2, Y1]; piecewise hyperbolic attractors [C1, Y1]; Hénon maps [BY, Y1]; non-uniformly expanding maps in one dimension [WY, Y1]; and Lorentz attractors [HM].

The strategy in all these papers is the same. One first constructs a Young tower $F: \Delta \circlearrowleft$ satisfying certain properties (see (P1)-(P5) of \$2.1). One then defines a quotient tower $\bar{\Delta}=\Delta / \sim$ where $x \sim y$ whenever $x$ and $y$ lie on the same stable leaf. There are thus three 
objects involved in the study of such systems. Letting $\pi$ and $\bar{\pi}$ represent the canonical projections from $(F, \Delta)$ to $(f, M)$ and $(\bar{F}, \bar{\Delta})$ respectively, we represent them as follows:

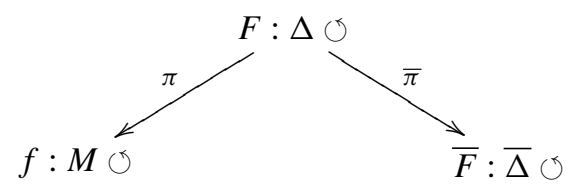

The reason for introducing the reduced tower $\bar{\Delta}$ is that since $\bar{F}: \bar{\Delta} \circlearrowleft$ is expanding, one can bring to bear the classical methods of analysis of the transfer operator for expanding maps. This functional analytic approach establishes the quasi-compactness of the transfer operator acting on certain spaces of functions and then links its peripheral spectrum to the statistical properties of the system $[\mathbf{B}, \mathbf{D F}, \mathbf{H H}, \mathbf{I M}, \mathbf{K}, \mathbf{L Y}, \mathbf{N}]$. In the case of Young towers, one must then pass these statistical properties from $\bar{\Delta}$ back up to $\Delta$ before projecting them down to $M$.

The problem is that in general one cannot lift measures from $\bar{\Delta}$ to $\Delta$ so that the usual procedure is to first prove the existence of an invariant measure on $\Delta$ separately (see [Y1]) and then pass from $\bar{\Delta}$ to $\Delta$ the desired properties related to the invariant measure such as central limit theorems, decay of correlations or large-deviation estimates (cf. [MN, RY]). From $\Delta$, these project easily onto $M$.

The purpose of this paper is to simplify the application of Young towers by directly studying the transfer operator $\mathcal{L}$ associated with $(F, \Delta)$, thus eliminating the need for the reduced tower $(\bar{F}, \bar{\Delta})$ entirely. We introduce Banach spaces on which $\mathcal{L}$ is quasicompact and obtain its spectral decomposition, following the recent extensions of this method to the hyperbolic setting [BT, BKL, DL, GL, R]. In doing so, we recover the statistical properties which have been proven for $f: M \circlearrowleft$ previously. In addition, we are able to obtain much more information about the evolution of non-invariant measures under the dynamics of $f$. We include as an example a large-deviation principle for a large class of initial distributions which is entirely new in the non-uniformly hyperbolic setting (Theorems 4 and 5) and include an explicit application to dispersing billiards (Theorem 6). We show that the rate function governing the large deviations is independent of the initial distribution.

It may be of some independent interest that when no contracting directions are present, i.e., when $f$ itself is expanding, the norms we define for hyperbolic towers reduce to norms for expanding towers which yield analogous results: i.e., the quasi-compactness of the transfer operator and a spectral gap. This is a characteristic of our norms which has not been present previously in the hyperbolic setting and which yields a unified treatment of hyperbolic and expanding Young towers (see Remark 2.2 for more details).

The rest of this paper is organized as follows. In $\S 2$, we define Young towers precisely, define the relevant norms and state our results. In $\$ 3$, we explore some properties of the Banach spaces while $\S \S 4$ and 5 contain the required Lasota-Yorke type estimates and spectral results. Large-deviation estimates and applications are proved in $\S \S 6$ and 7.

\section{Setting and statement of results}

2.1. Definition of the tower. We recall the definition of a Young tower $(F, \Delta)$ as described in [Y1]. We begin with a piecewise smooth map on a finite-dimensional 
Riemannian manifold, $f: M \circlearrowleft$, and let $\mu$ (respectively $\mu_{\gamma}$ ) denote Riemannian volume on $M$ (respectively $\gamma$ where $\gamma \subset M$ is a submanifold). We say that $f$ admits a generalized horseshoe if there exists a compact subset $\Lambda$ of $M$ satisfying properties (P1)-(P5) below. (We recall only the main properties; see [Y1] for more details.)

(P1) Hyperbolic product structure. $\Lambda=\bigcup_{j=1}^{J} \Lambda^{(j)}$ and each of the $\Lambda^{(j)}$ has the following product structure $\dagger: \Lambda^{(j)}=\left(\bigcup_{\gamma^{u} \in \Gamma_{j}^{u}} \gamma^{u}\right) \cap\left(\bigcup_{\gamma^{s} \in \Gamma_{j}^{s}} \gamma^{s}\right)$ where $\Gamma^{u}=\bigcup_{j} \Gamma_{j}^{u}$ and $\Gamma^{s}=\bigcup_{j} \Gamma_{j}^{s}$ are continuous families of local stable and unstable manifolds such that, for every $j$, each $\gamma^{u} \in \Gamma_{j}^{u}$ intersects every $\gamma^{s} \in \Gamma_{j}^{s}$ in a unique point. Moreover, $\mu_{\gamma}(\gamma \cap \Lambda)>0$ for each $\gamma \in \Gamma^{u}$.

A set $A$ is an $s$-subset (respectively $u$-subset) of $\Lambda$ if $\gamma \cap A \neq \emptyset$ implies $\gamma \subseteq A$ for any $\gamma \in \Gamma^{s(u)}$.

(P2) Return time function. Each $\Lambda^{(j)}$ is partitioned into countably many $s$-subsets $\Lambda_{i}^{(j)}$ with $\mu_{\gamma}\left(\Lambda^{(j)} \backslash \bigcup_{i} \Lambda_{i}^{(j)}\right)=0$ for all $\gamma \in \Gamma_{j}^{u}$. There exists a function, $R: \Lambda \rightarrow \mathbb{Z}^{+}$, constant on each $\Lambda_{i}^{(j)}$, such that $f^{R\left(\Lambda_{i}^{(j)}\right)}\left(\Lambda_{i}^{(j)}\right)$ is a $u$-subset of one of the $\Lambda^{(k)}$. Moreover, for each $n$, the number of $(i, j)$ such that $R_{i}^{(j)}=n$ is finite.

We refer to elements of $\Gamma^{u(s)}$ by $\gamma^{u(s)}$ and $J^{u} f$ denotes the unstable Jacobian of $f$ with respect to $\mu_{\gamma^{u}}$. Denote by $\gamma^{s}(x)$ and $\gamma^{u}(x)$ the stable and unstable leaves through $x$, respectively. For $x, y \in \Lambda$, there exists a separation time $s_{0}(x, y)$, depending only on the unstable coordinate, and numbers $C_{0} \geq 1, \alpha<1$ independent of $x, y$, such that the following hold.

(P3) Contraction on $\Gamma^{s}$. For $y \in \gamma^{s}(x), d\left(f^{n} x, f^{n} y\right) \leq C_{0} \alpha^{n} d(x, y)$ for all $n \geq 0$ ‡.

(P4) Backward contraction and distortion on $\Gamma^{u}$. Let $y \in \gamma^{u}(x)$ and $0 \leq k \leq n<$ $s_{0}(x, y)$.

(a) $\quad d\left(f^{n} x, f^{n} y\right) \leq C_{0} \alpha^{s_{0}(x, y)-n}$.

(b) $\log \prod_{i=k}^{n} J^{u} f\left(f^{i} x\right) / J^{u} f\left(f^{i} y\right) \leq C_{0} \alpha^{s_{0}(x, y)-n}$.

(P5) Convergence of $J^{u} f^{n}$ and absolute continuity of $\Gamma^{s}$.

(a) For $y \in \gamma^{s}(x)$ and all $n \geq 0$,

$$
\log \prod_{i=n}^{\infty} \frac{J^{u} f\left(f^{i} x\right)}{J^{u} f\left(f^{i} y\right)} \leq C_{0} \alpha^{n} .
$$

(b) Given $\gamma, \gamma^{\prime} \in \Gamma^{u}$, define $\Phi: \gamma \rightarrow \gamma^{\prime}$ by $\Phi(x)=\gamma^{s}(x) \cap \gamma^{\prime}$. Then $\Phi$ is absolutely continuous and

$$
\frac{d\left(\Phi_{*}^{-1} \mu_{\gamma^{\prime}}\right)}{d \mu_{\gamma}}=\prod_{i=0}^{\infty} \frac{J^{u} f\left(f^{i} x\right)}{J^{u} f\left(f^{i} \Phi x\right)} .
$$

The structure of the generalized horseshoe immediately yields the existence the Young tower,

$$
\Delta=\{(x, n) \in \Lambda \times \mathbb{N}: n<R(x)\} .
$$

$\dagger$ In [Y1], the tower has a single base $\Lambda$ and all returns are full returns to the base. In the present paper we treat the generalized case of towers with multiple bases. These are the $\Lambda^{(j)}$.

$\ddagger$ As an abstract requirement, (P3) is slightly stronger than the inequality $d\left(f^{n} x, f^{n} y\right) \leq C_{0} \alpha^{n}$ stated in [Y1]. In practice, however, the stronger version holds for all systems for which Young towers have been constructed to date. 
The tower map $F$ is defined by $F(x, \ell)=(x, \ell+1)$ for $\ell<R(x)-1$ and $F(x, R(x)-1)=\left(f^{R(x)} x, 0\right)$. We refer to $\Delta_{\ell}:=\left.\Delta\right|_{n=\ell}$ as the $\ell$ th level of the tower and to a point $(x, \ell)$ as simply $x$ where the level $\ell$ will be made clear by context.

$F$ admits a Markov partition $\left\{\Delta_{\ell, j}\right\}$ which has finitely many elements on each level $\ell$. We identify $\Delta_{0, j}$ with $\Lambda^{(j)}$ for $j=1, \ldots, J$. We define a separation time $s(x, y) \leq s_{0}(x, y)$ by $s(x, y)=\inf \left\{n>0: F^{n} x, F^{n} y\right.$ lie in different $\left.\Delta_{\ell, j}\right\}$. There is a canonical projection $\pi: \Delta \rightarrow M$ which conjugates the dynamics, $\pi \circ F=f \circ \pi . \pi$ is not necessarily one-to-one or onto, but if $f$ is injective, then so is $\left.\pi\right|_{\Delta_{\ell}}$ for each $\ell$.

We call $F$ transitive if, for all $j, j^{\prime}$, there exists $n \geq 0$ such that $F^{n}\left(\Delta_{0, j}\right) \cap \Delta_{0, j^{\prime}} \neq \emptyset$. We say $F$ is mixing if, for all $j, j^{\prime}$, there exists $N \geq 0$ such that $F^{n}\left(\Delta_{0, j}\right) \cap \Delta_{0, j^{\prime}} \neq \emptyset$ for all $n \geq N$. Similarly, we call $\mathcal{T} \subset \Delta$ a transitive component if $\mathcal{T}$ is a union of elements $\Delta_{\ell, j}$ such that $\left.F\right|_{\mathcal{T}}$ is transitive.

The base of the tower, $\Delta_{0}$, is identified with $\Lambda$ and inherits the structure of $\Gamma^{s}$ and $\Gamma^{u}$ as well as the measures $\mu$ and $\mu_{\gamma}$. The measure $\mu$ is extended to $\Delta_{\ell}, \ell>0$, by defining $\mu(A)=\mu\left(F^{-\ell} A\right)$ for all measurable $A \subset \Delta_{\ell}$. Thus $J F$, the Jacobian of $F$ with respect to $\mu$, satisfies $J F \equiv 1$ except at return times. $F$ enjoys properties (P3)-(P5) at return times due to the identity $J \pi(F) J F=J f(\pi) J \pi$ and the fact that $J \pi \equiv 1$ on $\Delta_{0}$.

Since $F: \Delta_{\ell} \rightarrow \Delta_{\ell+1}$ is rigid translation, the foliations $\Gamma^{s}$ and $\Gamma^{u}$ extend naturally to the entire tower, i.e., $\Delta_{\ell, j}$ has a product structure given by $F^{\ell}(S)$ where $S$ is an $s$ subrectangle in $\Delta_{0}$, and hence in $\Lambda$. We call these defining foliations $\Gamma^{s}(\Delta)$ and $\Gamma^{u}(\Delta) \dagger$.

We say the tower has exponential return times if the following holds:

there exist constants $c_{0}>0$ and $\theta<1$ such that $\mu(R>n)=\mu\left(\bigcup_{\ell \geq n} \Delta_{\ell}\right) \leq c_{0} \theta^{n}$.

Exponential return times are essential to our approach since we will prove the existence of a spectral gap which in turn implies exponential decay of correlations. Towers with polynomial return times have been shown to admit polynomial decay of correlations [Y2]. Accordingly, we will assume that (1) holds throughout this paper.

2.1.1. A reference measure on $\Delta$. In each $\Delta_{\ell, j}$ we choose a representative curve $\hat{\gamma} \in \Gamma^{u}\left(\Delta_{\ell, j}\right)$. For any $\gamma \in \Gamma^{u}\left(\Delta_{\ell, j}\right)$, let $\Phi_{\gamma, \hat{\gamma}}: \gamma \rightarrow \hat{\gamma}$ denote the holonomy map along $\Gamma^{s}$-leaves and let $J^{u} F$ denote the unstable Jacobian of $F$ with respect to $\mu_{\gamma}$. Then define $m_{\gamma}$ by $d m_{\gamma}=\phi d \mu_{\gamma}$ where

$$
\phi(x)=\prod_{i=0}^{\infty} \frac{J^{u} F\left(F^{i} x\right)}{J^{u} F\left(F^{i}\left(\Phi_{\gamma, \hat{\gamma}} x\right)\right)} .
$$

Given $\gamma^{\prime} \in \Gamma^{u}\left(\Delta_{0}\right)$, if $\gamma \in \Gamma^{u}\left(\Delta_{\ell, j}\right)$ satisfies $F(\gamma \cap S)=\gamma^{\prime}$ for some $s$-subrectangle $S$, then for $x \in \gamma \cap S$, define $J_{\gamma} F(x)=d\left(m_{\gamma^{\prime}} \circ F\right) / d m_{\gamma}$. Elsewhere on $\Delta, J_{\gamma} F \equiv 1$. Similarly, one defines $J_{\gamma} F^{R}(x)$ whenever $F^{R}(\gamma \cap S)=\gamma^{\prime}$ for some $s$-subrectangle $S$. For convenience, we restate Lemma 1 from [Y1], which summarizes the important properties of $m_{\gamma}$.

$\dagger$ For maps with singularities, the elements of $\Gamma^{s}(\Delta)$ and $\Gamma^{u}(\Delta)$ are actually positive-measure Cantor sets in real stable and unstable manifolds for $f$, as described in [Y1]. For simplicity, we shall refer to these Cantor sets as stable and unstable leaves throughout. 
LEMMA 2.1. [Y1] Let $\gamma, \gamma^{\prime} \in \Gamma^{u}\left(\Delta_{\ell, j}\right)$.

(1) Let $\Phi_{\gamma, \gamma^{\prime}}: \gamma \rightarrow \gamma^{\prime}$ be the holonomy map along $\Gamma^{s}$-leaves as above. Then $\Phi_{*} m_{\gamma}=$ $m_{\gamma^{\prime}}$.

(2) $J_{\gamma} F(x)=J_{\gamma^{\prime}} F(y)$, for all $x \in \gamma, y \in \gamma^{s}(x) \cap \gamma^{\prime}$.

(3) There exists $C_{1}>0$ such that for all $x, y \in \gamma$ with $s_{0}(x, y) \geq R(x)$,

$$
\left|\frac{J_{\gamma} F^{R}(x)}{J_{\gamma} F^{R}(y)}-1\right| \leq C_{1} \alpha^{s\left(F^{R} x, F^{R} y\right) / 2} .
$$

Moreover by $(P 5)(a), e^{-C_{0}} \leq \phi \leq e^{C_{0}}$.

On each $\Delta_{\ell, j}$, we define the measure $\mu^{s}$ on $\Gamma^{u}\left(\Delta_{\ell, j}\right)$ to be the factor measure of $\left.\mu\right|_{\Delta_{\ell, j}}$ on unstable leaves normalized so that $\mu^{s}\left(\Gamma^{u}\left(\Delta_{\ell, j}\right)\right)=1$. We define $m$ to be the measure with factor measure $\mu^{s}$ and measures $m_{\gamma}$ on unstable leaves. Notice that in any $\Delta_{\ell, j}$, Lemma 2.1(1) implies that $m_{\gamma}(S)=m(S)$ for any $s$-subset $S \subseteq \Delta_{\ell, j}$ and $\gamma \in \Gamma^{u}\left(\Delta_{\ell, j}\right)$. This feature of $m_{\gamma}$ implies that $m$ is a product measure on each $\Delta_{\ell, j}$. When disintegrating $m$, we maintain the convention that $\mu^{s}$ is normalized, but $m_{\gamma}$ is not.

2.1.2. Transfer operator. The primary object of interest in this paper is the transfer operator $\mathcal{L}$ associated with $F$. Before defining it, we introduce a class of functions.

We define a metric along stable leaves which makes the distance between unstable leaves uniform. Fix $x \in \Delta_{0}$ and let $y \in \gamma^{s}(x)$. Let $\Phi: \gamma^{u}(x) \rightarrow \gamma^{u}(y)$ be the sliding map along stable leaves. Define $d_{s}(x, y):=\sup _{z \in \gamma^{u}(x)} d(z, \Phi z)$. We extend this metric to $\Delta_{\ell}, \ell>1$, by setting $d_{s}\left(F^{\ell} x, F^{\ell} y\right)=\alpha^{\ell} d_{s}(x, y)$ for all $\ell<R(x)$ and $y \in \gamma^{s}(x)$. By (P3),

$$
d_{s}\left(F^{n} x, F^{n} y\right) \leq C_{0} \alpha^{n} d_{s}(x, y) \text { for all } n \geq 0 \text { whenever } y \in \gamma^{s}(x) .
$$

The class of test functions we use are required to be smooth along stable leaves only. Let $\mathcal{F}_{b}$ denote the set of bounded measurable functions on $\Delta$. For $\varphi \in \mathcal{F}_{b}$ and $0<r \leq 1$, define

$$
H_{s}^{r}(\varphi)=\sup _{\gamma^{s} \in \Gamma^{s}(\Delta)} H^{r}\left(\left.\varphi\right|_{\gamma^{s}}\right) \quad \text { where } H^{r}\left(\left.\varphi\right|_{\gamma^{s}}\right)=\sup _{x, y \in \gamma^{s}}|\varphi(x)-\varphi(y)| d_{s}(x, y)^{-r} .
$$

If $A$ is an $s$-subset of $\Delta$, we define $|\varphi|_{\mathcal{C}_{s}^{r}(A)}=\sup _{\gamma^{s} \subset A}|\varphi|_{\mathcal{C}^{0}\left(\gamma^{s}\right)}+H^{r}\left(\left.\varphi\right|_{\gamma^{s}}\right)$ and let $\mathcal{C}_{s}^{r}(A)=\left\{\varphi \in \mathcal{F}_{b}:|\varphi|_{\mathcal{C}_{s}^{r}(A)}<\infty\right\}$.

For $h \in\left(\mathcal{C}_{s}^{r}(\Delta)\right)^{\prime}$ an element of the dual of $\mathcal{C}_{s}^{r}(\Delta)$, the transfer operator $\mathcal{L}:\left(\mathcal{C}_{s}^{r}(\Delta)\right)^{\prime} \rightarrow$ $\left(\mathcal{C}_{s}^{r}(\Delta)\right)^{\prime}$ is defined by

$$
\mathcal{L} h(\varphi)=h(\varphi \circ F) \quad \text { for each } \varphi \in \mathcal{C}_{S}^{r}(\Delta) .
$$

When $h$ is a measure absolutely continuous with respect to the reference measure $m$, we shall call its $L^{1}(m)$ density $h$ as well. Hence $h(\varphi)=\int_{\Delta} h \varphi d m$. With this convention, $L^{1}(m) \subset\left(\mathcal{C}_{S}^{r}(\Delta)\right)^{\prime}$ and one can restrict $\mathcal{L}$ to $L^{1}(m)$. In this case,

$$
\mathcal{L}^{n} h(x)=\sum_{y \in F^{-n} x} h(y)\left(J_{m} F^{n}(y)\right)^{-1}
$$

for each $n \geq 0$ where $J_{m} F^{n}$ is the Jacobian of $F^{n}$ with respect to $m$.

Along unstable leaves, we define the metric $d_{u}(x, y)=\beta_{0}^{s(x, y)}$ for $y \in \gamma^{u}(x)$ and some $\beta_{0}<1$ to be chosen later. Let $\operatorname{Lip}^{u}\left(\left.\varphi\right|_{\gamma}\right)$ denote the Lipschitz constant of a function $\varphi$ along $\gamma \in \Gamma^{u}$ with respect to $d_{u}$ and define $\operatorname{Lip}^{u}(\varphi)=\sup _{\gamma \in \Gamma^{u}(\Delta)} \operatorname{Lip}^{u}\left(\left.\varphi\right|_{\gamma}\right)$. We define $\operatorname{Lip}^{u}(\Delta)=\left\{\varphi \in \mathcal{F}_{b}: \operatorname{Lip}^{u}(\varphi)<\infty\right\}$. 
2.2. Definition of norms. Let $\mathcal{P}=\left\{\Delta_{\ell, j}\right\}$ denote the Markov partition for $F$. For each $k \geq 0$, define $\mathcal{P}^{k}=\bigvee_{i=0}^{k} F^{-i} \mathcal{P}$ and let $\mathcal{P}_{\ell, j}^{k}=\mathcal{P}^{k} \mid \Delta_{\ell, j}$. The elements $E \in \mathcal{P}_{\ell, j}^{k}$ are $k$-cylinders which are $s$-subsets of $\Delta_{\ell, j}$. For $\psi \in L^{1}(m)$ and $E \in \mathcal{P}^{k}$, define

$$
f_{E} \psi d m=\frac{1}{m(E)} \int_{E} \psi d m .
$$

Now choose $0<q<p \leq 1$ and fix $1>\beta_{0}>\max \{\theta, \sqrt{\alpha}\}$ where $\theta$ is given by (1) and $\alpha$ is from (P3). Next, choose $1>\beta \geq \max \left\{\beta_{0}^{(p-q) / p}, \alpha^{q}\right\}$.

For $h \in \operatorname{Lip}^{u}(\Delta)$, define the weak norm of $h$ by $|h|_{w}=\sup _{\ell, j, k}|h|_{w\left(\mathcal{P}_{\ell, j}^{k}\right)}$ where

$$
|h|_{w\left(\mathcal{P}_{\ell, j}^{k}\right)}=\beta_{0}^{\ell} \sup _{E \in \mathcal{P}_{\ell, j}^{k}|\varphi|_{\mathcal{C}_{s}^{p}(E)} \leq 1} \sup _{E} h \varphi d m .
$$

Define the strong stable norm of $h$ by $\|h\|_{s}=\sup _{\ell, j, k}\|h\|_{s\left(\mathcal{P}_{\ell, j}^{k}\right)}$ where

$$
\|h\|_{s\left(\mathcal{P}_{\ell, j}^{k}\right)}=\beta^{\ell} \sup _{E \in \mathcal{P}_{\ell, j}^{k}|\varphi|_{\mathcal{C}_{s}^{q}(E)} \leq 1} \sup _{E} h \varphi d m .
$$

For $\varphi \in \mathcal{C}_{s}^{p}(\Delta)$, define $\varphi_{E}$ on $E \in \mathcal{P}_{\ell, j}^{k}$ by $\varphi_{E}(x)=m(E)^{-1} \int_{\gamma^{u}(x) \cap E} \varphi d m_{\gamma}$, for $x \in E$. Let $\tilde{\varphi}_{E}(x)=\varphi_{E}\left(\gamma^{u}(x)\right)$ for $x \in \Delta_{\ell, j}$ be the extension of $\varphi_{E}$ to $\Delta_{\ell, j}$. Note that $\tilde{\varphi}_{E}$ is welldefined since $\varphi_{E}$ is constant on unstable leaves. In what follows, let $E_{k} \in \mathcal{P}_{\ell, j}^{k}, E_{r} \in \mathcal{P}_{\ell, j}^{r}$ for $r \geq k$.

We define the strong unstable norm of $h$ by $\|h\|_{u}=\sup _{\ell, j, k}\|h\|_{u\left(\mathcal{P}_{\ell, j}^{k}\right)}$ where

$$
\|h\|_{u\left(\mathcal{P}_{\ell, j}^{k}\right)}=\sup _{E_{k} \in \mathcal{P}_{\ell, j}^{k}} \sup _{E_{r} \subset E_{k}|\varphi|_{\mathcal{C}_{S}\left(E_{r}\right)}^{p} \leq 1} \sup \beta^{\ell-k}\left|f_{E_{r}} h \varphi d m-f_{E_{k}} h \tilde{\varphi}_{E_{r}} d m\right| .
$$

The strong norm of $h$ is defined as $\|h\|=\|h\|_{s}+b\|h\|_{u}$, for some $b>0$ to be chosen later.

We denote by $\mathcal{B}$ the completion of $\operatorname{Lip}^{u}(\Delta)$ in the $\|\cdot\|$-norm and by $\mathcal{B}_{w}$ the completion of $\operatorname{Lip}^{u}(\Delta)$ in the $|\cdot|_{w}$ norm.

Remark 2.2. If there is no stable direction, on each $\Delta_{\ell, j}$ the weak norm, $|\cdot|_{w}$, reduces to the $\mathcal{C}^{0}$ norm of $h$ weighted by $\beta_{0}^{\ell}$. Similarly, the strong stable and unstable norms reduce to the $\mathcal{C}^{0}$ norm and Lipschitz constant of $h$ respectively, each weighted by $\beta^{\ell}$. The LasotaYorke estimates (Proposition 2.3) and the compactness argument (Lemma 3.6) both hold in this setting so that one immediately obtains the results of Theorem 1.

2.3. Statement of results on the tower. The standing assumptions throughout this paper are that $(F, \Delta)$ satisfies $(\mathrm{P} 1)-(\mathrm{P} 5)$ and $(1)$.

Proposition 2.3. There exists $C>0$ such that, for each $h \in \mathcal{B}$ and $n \geq 0$,

$$
\begin{gathered}
\left|\mathcal{L}^{n} h\right|_{w} \leq C|h|_{w}, \\
\left\|\mathcal{L}^{n} h\right\|_{s} \leq C \beta^{n}\|h\|_{s}+C|h|_{w}, \\
\left\|\mathcal{L}^{n} h\right\|_{u} \leq C \beta^{n}\|h\|_{u}+C\|h\|_{s} .
\end{gathered}
$$


For any $1>\tau>\beta$, there exists $N \geq 0$ such that $2 C \beta^{N}<\tau^{N}$. Choose $b=\beta^{N}$. Then,

$$
\begin{aligned}
\left\|\mathcal{L}^{N} h\right\| & =\left\|\mathcal{L}^{N} h\right\|_{s}+b\left\|\mathcal{L}^{N} h\right\|_{u} \\
& \leq C \beta^{N}\left(\|h\|_{s}+b\|h\|_{u}\right)+b C\|h\|_{s}+C|h|_{w} \\
& \leq \tau^{N}\|h\|+C|h|_{w} .
\end{aligned}
$$

The above represents the traditional Lasota-Yorke inequality. Since by Lemma 3.6, the unit ball of $\mathcal{B}$ is relatively compact in $\mathcal{B}_{w}$, it follows from standard arguments $[\mathbf{D F}, \mathbf{B}]$ that the essential spectral radius of $\mathcal{L}$ on $\mathcal{B}$ is bounded by $\beta$. Our first theorem presents the decomposition of the peripheral spectrum.

THEOREM 1. The operator $\mathcal{L}: \mathcal{B} \circlearrowleft$ is quasi-compact with essential spectral radius $\beta$ and spectral radius 1 . In addition, the following hold.

(i) If $F$ is mixing, then 1 is a simple eigenvalue and all other eigenvalues have modulus strictly less than 1 .

(ii) If $F$ is transitive and periodic with period $p$, then the set of eigenvalues of modulus 1 consists of simple eigenvalues $\left\{e^{2 \pi i k / p}\right\}_{k=0}^{p-1}$.

(iii) In general, $F$ has finitely many transitive components, each with largest eigenvalue

1. On each component, (ii) applies.

Let $V_{\phi}$ be the eigenspace of $\mathcal{L}$ corresponding to the eigenvalue $e^{i \phi}$ and set $V:=\oplus_{\phi} V_{\phi}$. Our next results characterize the set of invariant measures in $\mathcal{B}$ and some of the statistical properties of $F$. Let $\mathcal{C}_{b}^{0}(\Delta)$ denote the set of bounded functions on $\Delta$ which are continuous on each $\Delta_{\ell, j}$. Recall that an invariant probability measure $v$ is called a physical measure if there exists a positive Lebesgue measure invariant set $B_{v}$, with $v\left(B_{v}\right)=1$, such that, for each $\psi \in \mathcal{C}_{b}^{0}$,

$$
\lim _{n \rightarrow \infty} \frac{1}{n} \sum_{i=0}^{n-1} \psi\left(F^{i} x\right)=v(\psi) \quad \text { for all } x \in B_{\nu} .
$$

THEOREM 2.

(i) Each $v \in V$ is a signed measure absolutely continuous with respect to the probability measure $\bar{v}:=\lim _{n \rightarrow \infty}(1 / n) \sum_{i=0}^{n-1} \mathcal{L}^{i} 1$. The conditional measures of $v$ on $\gamma \in \Gamma^{u}$ are absolutely continuous with respect to $\mu_{\gamma}$.

(ii) F admits only finitely many physical measures and they are precisely the ergodic elements of $V_{0}$. The supports of the physical measures correspond to the ergodic decomposition with respect to Lebesgue and $\bar{v}(\mathcal{E})=m(\mathcal{E})$ for each ergodic component $\mathcal{E}$.

(iii) For all $\psi \in \mathcal{C}_{b}^{0}(\Delta)$ and every $\gamma \in \Gamma^{u}(\Delta)$, the limit $\psi^{+}(x):=\lim _{n \rightarrow \infty}(1 / n) \sum_{i=0}^{n-1} \psi$ $\circ F^{i}(x)$ exists for $\mu_{\gamma}$-almost every $x \in \gamma$ and takes on only finitely many different values in $\Delta$. If $\bar{v}$ is ergodic, then $\psi^{+}(x)=\int \psi d \bar{v}$ for $\mu_{\gamma}$-almost every $x$.

(iv) If $F$ is mixing, then $F$ exhibits exponential decay of correlations for Hölder observables, and the central limit theorem holds. In particular, there exist constants $C_{1}>0, \sigma<1$, such that for any $\psi \in \operatorname{Lip}^{u}(\Delta)$ and $\varphi \in \mathcal{C}_{s}^{p}(\Delta)$,

$$
\left|\int \psi \varphi \circ F^{n} d \bar{v}-\bar{v}(\psi) \bar{v}(\varphi)\right| \leq C_{1} \sigma^{n}|\varphi|_{\mathcal{C}_{s}^{p}(\Delta)}\left(|\psi|_{\infty}+\operatorname{Lip}^{u}(\psi)\right) .
$$


2.3.1. Large-deviation estimates. An immediate application of the spectral decomposition of $\mathcal{L}$ is the derivation of large-deviation estimates for smooth observables $g: \Delta \rightarrow \mathbb{R}$.

Let $f: X \circlearrowleft$ be a measurable map. Letting $S_{n} g=\sum_{k=0}^{n-1} g \circ f^{k}$, if $v$ is an ergodic invariant measure, then $(1 / n) S_{n}(g)$ converges to $v(g)$ by the Birkhoff ergodic theorem. Large-deviation estimates provide exponential bounds on the rate of convergence of $S_{n} g$ to the mean $v(g)$. These typically take the form

$$
\lim _{\varepsilon \rightarrow 0} \lim _{n \rightarrow \infty} v\left(x \in \Delta: \frac{1}{n} S_{n}(g)(x) \in[t-\varepsilon, t+\varepsilon]\right)=-I(t)
$$

where $I(t) \geq 0$ is called the rate function.

Large-deviation estimates of this type have been proved for systems admitting Young towers [RY, MN]. When $f$ is (non-uniformly) expanding and $v$ is has strictly positive density with respect to Lebesgue, the measure on the left-hand side of (9) can be replaced by Lebesgue [KN]. This is also true when $f$ is Axiom A [OP]. For more general nonuniformly hyperbolic systems, (9) is not known to be true when $v$ is not an invariant measure.

In the setting of the present paper, we prove as a direct corollary of our operator approach that (9) holds for all probability measures in $\mathcal{B}$ with the same rate function $I$. Let $\sigma^{2}$ denote the limit as $n \rightarrow \infty$ of the variance of $(1 / \sqrt{n}) S_{n} g$ with respect to the unique invariant measure for $F$.

THEOREM 3. Let $(F, \Delta)$ be mixing and let $g \in \operatorname{Lip}^{u}(\Delta) \cap \mathcal{C}_{s}^{p}(\Delta)$. There exist constants $\tau_{\max }, \omega_{\max }>0$ such that for all probability measures $\eta \in \mathcal{B}$, the logarithmic moment generating function

$$
q(z)=\lim _{n \rightarrow \infty} \frac{1}{n} \log \eta\left(e^{z S_{n} g}\right)
$$

exists, is independent of $\eta$ and is analytic in the rectangle $\left\{z \in \mathbb{C}:|\operatorname{Re} z|<\tau_{\max },|\operatorname{Im} z|<\right.$ $\left.\omega_{\max }\right\}$. Moreover, $q^{\prime}(0)=\bar{v}(g), q^{\prime \prime}(0)=\sigma^{2}$ and $q(z)$ is strictly convex for real $z$ whenever $\sigma^{2}>0$.

An immediate consequence of this theorem is a large-deviation result for probability measures in $\mathcal{B}$.

THEOREM 4. Let $\eta \in \mathcal{B}$ be a probability measure and let $I(u)$ be the Legendre transform of $q(z)$. Then, for any interval $[a, b] \subset\left[q^{\prime}\left(-\tau_{\max }\right), q^{\prime}\left(\tau_{\max }\right)\right]$,

$$
\lim _{n \rightarrow \infty} \frac{1}{n} \log \eta\left(x \in \Delta: \frac{1}{n} S_{n} g(x) \in[a, b]\right)=-\inf _{u \in[a, b]} I(u) .
$$

2.4. Discussion of applications. Throughout this section, $f: M \circlearrowleft$ is a map which admits a Young tower $F: \Delta \circlearrowleft$ as described in $\$ 2.1$ with exponential return times. Let $\Gamma^{s(u)}(M)$ denote the set of local stable (unstable) manifolds on $M$. For $\zeta \geq 0, \mathcal{C}^{\zeta}\left(\Gamma^{u}(M)\right.$ ) denotes the set of functions $\varphi$ on $M$ which satisfy $\sup _{\gamma \in \Gamma^{u}(M)}|\varphi|_{\mathcal{C}^{\zeta}(\gamma)}<\infty$, and similarly for $\mathcal{C}^{\zeta}\left(\Gamma^{S}(M)\right)$. 
THEOREM 5.

(i) The map $f$ has at least one and at most finitely many physical measures which lift to $\Delta$. Each of these physical measures has absolutely continuous conditional measures on unstable leaves.

For (ii)-(iv), we assume $f$ is mixing and that $\xi \in \pi_{*} \mathcal{B}$ is a probability measure.

(ii) The map $f$ has a unique physical measure $\tilde{v}=\pi_{*} \bar{\nu}$. $\tilde{v}$ satisfies the central limit theorem and enjoys exponential decay of correlations, i.e., there exists $C_{2}>0$ such that

$$
\left|\int \psi \varphi \circ f^{n} d \tilde{v}-\tilde{v}(\psi) \tilde{v}(\phi)\right| \leq C_{2} \sigma^{n}|\psi|_{\mathcal{C}^{\zeta}\left(\Gamma^{u}(M)\right)}|\varphi|_{\mathcal{C}^{\zeta}\left(\Gamma^{s}(M)\right)}
$$

for all $\psi \in \mathcal{C}^{\zeta}\left(\Gamma^{u}(M)\right), \varphi \in \mathcal{C}^{\zeta}\left(\Gamma^{s}(M)\right)$, with $\sigma$ as in Theorem 2(iv).

(iii) The limit $\lim _{n \rightarrow \infty} f_{*}^{n} \xi=\tilde{v}$ weakly.

(iv) Suppose $\tilde{g} \in \mathcal{C}^{\zeta}(M)$ for some $\zeta>0$ and let $S_{n} \tilde{g}=\sum_{i=0}^{n-1} \tilde{g} \circ f^{i}$. Then the logarithmic moment generating function $q(z)=\lim _{n \rightarrow \infty}(1 / n) \log \xi\left(e^{S_{n} \tilde{g}}\right)$ exists and satisfies the conclusions of Theorem 3. As a consequence, all probability measures $\xi \in \pi_{*} \mathcal{B}$ satisfy the large-deviation principle given by Theorem 4 with the same rate function $I$.

The discussion of which measures on $M$ lift to $\Delta$, i.e., are in $\pi_{*} \mathcal{B}$, depends on the properties of the underlying system $(f, M)$. For non-uniformly expanding systems, one can show that Lebesgue measure $\mu \in \pi_{*} \mathcal{B}$ and indeed $\mathcal{C}^{\zeta}(M) \subset \pi_{*} \mathcal{B}$ under fairly mild assumptions (cf. [BDM]). For hyperbolic systems, the question is more subtle. One can easily see that measures supported on $\pi\left(\Delta_{\ell, j}\right)$ for finitely many $\Delta_{\ell, j}$ with smooth conditional densities on $\Gamma^{u}(M)$ lift to measures in $\mathcal{B}$. One may also allow singular measures supported on a single local unstable leaf. For more general measures the story is not so simple and in general one cannot even guarantee that Lebesgue measure lifts to $\Delta$. One class of measures which always lift to $\Delta$ are those measures which satisfy $\xi=\psi \tilde{v}$ for some $\psi \in \mathcal{C}^{\zeta}\left(\Gamma^{u}(M)\right)$, for then defining $\eta:=(\psi \circ \pi) \bar{\nu}$, one has $\eta \in \mathcal{B}$ and $\pi_{*} \eta=\xi$ (see, for example, [DWY, Lemma 6.3]).

For systems whose invariant measure is smooth with respect to Lebesgue, such as dispersing billiards, we obtain convergence results for a class of measures which do not lift to $\Delta$, but can be approximated by elements of $\pi_{*} \mathcal{B}$.

THEOREM 6. Let $(f, M)$ be the billiard map corresponding to a dispersing billiard which admits a mixing Young tower with exponential tail bounds as in [Y1, C3]. Let $\mu$ be Lebesgue measure and let $v$ be the smooth invariant measure for $f$. Let $\tilde{\mathcal{G}}$ denote the set of probability measures $\xi$ on $M$ such that $d \xi / d \mu \in \mathcal{C}^{\zeta}\left(\Gamma^{u}(M)\right)$ for some $\zeta>0$. Let $\xi \in \tilde{\mathcal{G}} \cup \pi_{*} \mathcal{B}$ be a probability measure. Then the following hold.

(i) Convergence to $v$. $f_{*}^{n} \xi$ converges weakly to $v$ as $n \rightarrow \infty$.

(ii) Large deviations. For any $g \in \mathcal{C}^{\zeta}(M)$, $\xi$ satisfies the large-deviation estimate of Theorem 4 with rate function I independent of $\xi$.

3. Properties of the spaces $\mathcal{B}$ and $\mathcal{B}_{w}$

Before exploring the generalized function spaces we have defined, we record the following lemma for future use. 
LeMma 3.1. (Regularity of $\varphi_{E}$ ) Let $0<r \leq 1$ and $k \geq 0$. For $E \in \mathcal{P}_{\ell, j}^{k}$ and $\varphi \in \mathcal{C}_{s}^{r}(E)$, let $\varphi_{E}$ be defined as in $\$ 2.2$. Then $\varphi_{E} \in \mathcal{C}_{S}^{r}(E)$ and $\left|\varphi_{E}\right|_{\mathcal{C}_{s}^{r}(E)} \leq|\varphi|_{\mathcal{C}_{s}^{r}(E)}$.

Proof. Choose $E \in \mathcal{P}_{\ell, j}^{k}$ and $\varphi \in \mathcal{C}_{S}^{r}(E)$. By definition, $\varphi_{E}(x)=m(E)^{-1} \int_{\gamma^{u}(x) \cap E} \varphi d m_{\gamma}$. This immediately implies $\left|\varphi_{E}\right|_{\infty} \leq|\varphi|_{\infty}$.

Take $x, y \in \gamma^{s} \subset E$ and let $\Phi: \gamma^{u}(x) \rightarrow \gamma^{u}(y)$ denote the holonomy map along stable leaves. Note that $J \Phi \equiv 1$ by Lemma 2.1(1). Then

$$
\left|\varphi_{E}(x)-\varphi_{E}(y)\right| \leq m(E)^{-1}\left|\int_{\gamma^{u}(x) \cap E}(\varphi-\varphi \circ \Phi) d m_{\gamma}\right| \leq H_{s}^{r}(\varphi) d_{s}(x, y)^{r},
$$

using the fact that $m_{\gamma}(E)=m(E)$ and $d_{s}(z, \Phi z)=d_{s}(x, y)$ for all $z \in \gamma^{u}(x)$.

\subsection{Embeddings.}

LEMMA 3.2. There exists $C>0$ such that for all $h \in \mathcal{B}_{w}$ and $\varphi \in \mathcal{C}_{s}^{p}(\Delta)$, we have

$$
|h(\varphi)| \leq C|h|_{w}\left(|\varphi|_{\infty}+H_{s}^{p}(\varphi)\right) .
$$

Proof. Let $h \in \operatorname{Lip}^{u}(\Delta)$ and $\varphi \in \mathcal{C}_{s}^{p}(\Delta)$. Then

$$
\begin{aligned}
\int_{\Delta} h \varphi d m & =\sum_{\ell, j} \int_{\Delta_{\ell, j}} h \varphi d m \leq \sum_{\ell, j} m\left(\Delta_{\ell, j}\right) \beta_{0}^{-\ell}|h|_{w}|\varphi|_{\mathcal{C}_{s}^{p}\left(\Delta_{\ell, j}\right)} \\
& \leq C|h|_{w}\left(|\varphi|_{\infty}+H_{s}^{p}(\varphi)\right),
\end{aligned}
$$

since $\beta_{0}>\theta$ and $m\left(\Delta_{\ell, j}\right) \leq e^{C_{0}} \mu\left(\Delta_{\ell, j}\right)$ by Lemma 2.1 .

It is clear from the definition of $\|\cdot\|_{u}$ that measures $h \in \mathcal{B}$ necessarily have absolutely continuous conditional measures on $h$-almost-every $\gamma \in \Gamma^{u}(\Delta)$. Lemma 3.3 provides some examples of what types of measures are found in $\mathcal{B}$.

Given a measure $\eta$ with absolutely continuous conditional measures on unstable leaves, we define a measure $\eta^{s}$ on $\Gamma^{u}(\Delta)$, i.e., a measure transverse to unstable leaves, as follows: set $\eta^{s}\left(\Gamma^{u}\left(\Delta_{\ell, j}\right)\right)=0$ if $\left.\eta\right|_{\Delta_{\ell, j}} \equiv 0$. If $\left.\eta\right|_{\Delta_{\ell, j}} \neq 0$, then $\left.\eta^{s}\right|_{\Gamma^{u}\left(\Delta_{\ell, j}\right)}$ is the factor measure of $\left.\eta\right|_{\Delta_{\ell, j}}$ normalized, and $\left\{\rho_{\gamma} d m_{\gamma}, \gamma \in \Gamma^{u}\left(\Delta_{\ell, j}\right)\right\}$ is the disintegration of $\eta$ into measures on unstable leaves. We will use the convention that $\eta^{s}\left(\Delta_{\ell, j}\right)=1$, and that the densities $\rho_{\gamma}$ are not normalized.

We define $\mathcal{G}$ to be the set of such measures $\eta \neq 0$ whose (unnormalized) densities satisfy $\sup _{\gamma \in \Gamma^{u}(\Delta)}\left|\rho_{\gamma}\right|_{C^{0}(\gamma)}+\operatorname{Lip}^{u}\left(\rho_{\gamma}\right)<\infty$.

LEMMA 3.3.

(i) $\mathcal{G} \subset \mathcal{B}$ and in particular $\operatorname{Lip}^{u}(\Delta) \subset \mathcal{B}$.

(ii) If $h \in \operatorname{Lip}^{u}(\Delta) \cap \mathcal{C}_{s}^{p}(\Delta)$ and $g \in \mathcal{B}$, then $h g \in \mathcal{B}$,

$$
\|h g\|_{s} \leq\|g\|_{s}|h|_{\mathcal{C}_{s}^{q}(\Delta)} \quad \text { and } \quad\|h g\|_{u} \leq\|g\|_{s}\left(\operatorname{Lip}^{u}(h)+H_{S}^{p}(h)\right)+\|g\|_{u}|h|_{\mathcal{C}_{s}^{p}(\Delta)} .
$$

Proof. (i) We first show $\operatorname{Lip}^{u}(\Delta) \subset \mathcal{B}$ and then use these functions to approximate singular measures $\eta$ in the $\|\cdot\|$-norm. Let $h \in \operatorname{Lip}^{u}(\Delta)$. It follows immediately that $\|h\|_{s} \leq|h|_{\infty}$. 
Now let $E_{k} \in \mathcal{P}_{\ell, j}^{k}$ and take $E_{r} \in \mathcal{P}_{\ell, j}^{r}$ with $E_{r} \subseteq E_{k}$. For $\varphi \in \mathcal{C}_{s}^{p}\left(E_{r}\right)$, define $\tilde{\varphi}_{E_{r}}$ as in $\S 2.2$. Choose $\gamma_{0}^{s} \in \Gamma^{s}\left(E_{k}\right)$ and let $h_{0}(x)=h\left(\gamma^{u}(x) \cap \gamma_{0}^{s}\right)$ for $x \in \Delta_{\ell, j}$. Then

$$
\begin{aligned}
f_{E_{r}} h \varphi d m-f_{E_{k}} h \tilde{\varphi}_{E_{r}} d m= & f_{E_{r}}\left(h-h_{0}\right) \varphi d m+f_{E_{r}} h_{0} \varphi d m \\
& -f_{E_{k}} h_{0} \tilde{\varphi}_{E_{r}} d m+f_{E_{k}}\left(h_{0}-h\right) \tilde{\varphi}_{E_{r}} d m .
\end{aligned}
$$

The first term of (10) is $\leq \operatorname{Lip}^{u}(h) \beta^{k}|\varphi|_{\mathcal{C}_{s}^{q}\left(E_{r}\right)}$. The fourth term has the same bound since $\left|\tilde{\varphi}_{E_{r}}\right|_{\mathcal{C}_{s}^{q}\left(E_{k}\right)} \leq|\varphi|_{\mathcal{C}_{s}^{q}\left(E_{r}\right)}$ by Lemma 3.1. Note that by Lemma 2.1(1), $m_{\gamma}\left(\gamma \cap E_{k}\right)=m\left(E_{k}\right)$ for each $\gamma \in \Gamma^{u}\left(\Delta_{\ell, j}\right)$ since $\mu^{s}$ is a probability measure. Since $h_{0}$ and $\tilde{\varphi}_{E_{r}}$ are both constant on unstable leaves,

$$
f_{E_{k}} h_{0} \tilde{\varphi}_{E_{r}} d m=m\left(E_{k}\right)^{-1} \int_{\Gamma^{u}\left(\Delta_{\ell, j}\right)} d \mu^{s}(\gamma) h_{0} \tilde{\varphi}_{E_{r}} m_{\gamma}\left(E_{k}\right)=f_{E_{r}} h_{0} \varphi d m,
$$

so that the second and third terms of (10) cancel. Thus $\|h\|_{u} \leq 2 \operatorname{Lip}^{u}(h)$ and $h \in \mathcal{B}$.

To prove the result for more general $\eta \in \mathcal{G}$, it suffices to prove it for measures supported on a single unstable leaf. More general measures follow by approximation.

Fix $\gamma_{0} \in \Gamma^{u}\left(\Delta_{\ell, j}\right)$ and let $\rho$ be a Lipschitz function on $\gamma_{0}$. Let $\eta=\rho m_{\gamma_{0}}$. We take a sequence of smooth functions $\psi_{n}$, depending only on the stable coordinate, which converge in distribution to $\delta_{\gamma_{0}}$. Extend $\rho$ to $\Delta_{\ell, j}$ by making it constant on stable leaves and define $h_{n}=\psi_{n} \rho$. Note that $h_{n} \in \operatorname{Lip}^{u}(\Delta) \cap \mathcal{C}_{s}^{p}(\Delta)$ and $h_{n}$ clearly converges to $\eta$ in distribution. It remains to show that $\left\{h_{n}\right\}$ is a Cauchy sequence in the $\|\cdot\|$-norm.

For $E \in \mathcal{P}_{\ell, j}^{k}$ and $\varphi \in \mathcal{C}_{s}^{q}(E)$,

$$
f_{E}\left(h_{n}-h_{m}\right) \varphi d m=\frac{1}{m(E)} \int_{\Gamma^{u}\left(\Delta_{\ell, j}\right)} d \mu^{s}(\gamma)\left(\psi_{n}(\gamma)-\psi_{m}(\gamma)\right) \int_{\gamma \cap E} \rho \varphi d m_{\gamma} .
$$

Since $m(E)^{-1} \int_{\gamma \cap E} \rho \varphi d m_{\gamma}$ is a Hölder continuous function of $\gamma$ with $\mathcal{C}^{q}$ norm bounded by $|\rho|_{\infty}|\varphi|_{\mathcal{C}_{s}^{q}(E)}$, the integral above converges to 0 as $n, m \rightarrow \infty$. Moreover, since the $\mathcal{C}^{q}$ norms are uniformly bounded for $|\varphi|_{\mathcal{C}_{S}^{q}(E)} \leq 1$, we may take the supremum and conclude convergence in the $\|\cdot\|_{s}$-norm.

To estimate $\left\|h_{n}-h_{m}\right\|_{u}$, let $E_{r} \subseteq E_{k} \subseteq \Delta_{\ell, j}$ and for $\varphi \in \mathcal{C}_{s}^{p}\left(\Delta_{\ell, j}\right)$, define $\tilde{\varphi}_{E_{r}}$ as in $\S 2.2$. Choose $\gamma^{s} \in \Gamma^{s}\left(E_{r}\right)$ and let $\bar{\rho}(x)=\rho\left(\gamma^{u}(x) \cap \gamma^{s}\right)$ for $x \in \Delta_{\ell, j}$. Now set $g_{n, m}=h_{n}-h_{m}$ and $\bar{g}_{n, m}=\left(\psi_{n}-\psi_{m}\right) \bar{\rho}$. Then following (10), since $\bar{g}_{n, m}$ is constant along unstable leaves, we have

$$
f_{E_{r}} g_{n, m} \varphi d m-f_{E_{k}} g_{n, m} \tilde{\varphi}_{E_{r}} d m=f_{E_{r}}\left(g_{n, m}-\bar{g}_{n, m}\right) \varphi d m+f_{E_{k}}\left(\bar{g}_{n, m}-g_{n, m}\right) \tilde{\varphi}_{E_{r}} d m \text {. }
$$

The first term above is equal to $m\left(E_{r}\right)^{-1} \int d \mu^{s}(\gamma)\left(\psi_{n}-\psi_{m}\right) \int_{\gamma \cap E_{r}}(\rho-\bar{\rho}) \varphi d m_{\gamma}$. Dividing by $\beta^{k}$ and using the fact that $\beta^{-k}(\rho-\bar{\rho})$ is bounded on $E_{r}$ since $\rho \in \operatorname{Lip}^{u}\left(\Delta_{\ell, j}\right)$, we see that the integral of interest has the form $\int d \mu^{s}(\gamma)\left(\psi_{n}-\psi_{m}\right) \Psi$, where $\Psi$ is a Hölder continuous function of $\gamma$ with Hölder norm bounded by $\operatorname{Lip}^{u}(\rho)|\varphi|_{\mathcal{C}_{s}^{p}\left(\Delta_{\ell, j}\right)}$. We conclude that the integral converges to zero as $n, m \rightarrow \infty$, uniformly for $|\varphi|_{\mathcal{C}_{s}^{p}\left(\Delta_{\ell, j}\right)} \leq 1$. A similar estimate holds for the second term of (11) so that $\left\|h_{n}-h_{m}\right\|_{u} \rightarrow 0$ as $n, m \rightarrow \infty$. 
(ii) By density of $\operatorname{Lip}^{u}(\Delta) \subset \mathcal{B}$, it suffices to prove the claim for $h \in \operatorname{Lip}^{u}(\Delta) \cap \mathcal{C}_{s}^{p}(\Delta)$ and $g \in \operatorname{Lip}^{u}(\Delta)$. That $\|h g\|_{s} \leq\|g\|_{s}|h|_{\mathcal{C}_{s}^{q}(\Delta)}$ is immediate.

Let $E_{r} \subseteq E_{k}, \varphi \in \mathcal{C}_{s}^{p}(\Delta)$ and $\tilde{\varphi}_{E_{r}}$ be as above. We define $h_{0}$ as in (i) and follow (10) to obtain

$$
\begin{aligned}
f_{E_{r}} h g \varphi d m-f_{E_{k}} g h \tilde{\varphi}_{E_{r}} d m= & f_{E_{r}}\left(h-h_{0}\right) g \varphi d m+f_{E_{r}} h_{0} g \varphi d m \\
& -f_{E_{k}} h_{0} g \tilde{\varphi}_{E_{r}} d m+f_{E_{k}}\left(h_{0}-h\right) g \tilde{\varphi}_{E_{r}} d m .
\end{aligned}
$$

To estimate the second and third terms of (12), we use the fact that $h_{0} \in \mathcal{C}_{s}^{p}\left(\Delta_{\ell, j}\right)$ and $\left(h_{0} \varphi\right)_{E_{r}}=h_{0} \varphi_{E_{r}}$ since $h_{0}$ is already constant on unstable leaves, where $\left(h_{0} \varphi\right)_{E_{r}}$ denotes the average of $h_{0} \varphi$ on unstable leaves in $E_{r}$. Then

$$
f_{E_{r}} g h_{0} \varphi d m-f_{E_{k}} g h_{0} \tilde{\varphi}_{E_{r}} d m \leq \beta^{k-\ell}\|g\|_{u}|h|_{\mathcal{C}_{s}^{p}\left(\Delta_{\ell, j}\right)}|\varphi|_{\mathcal{C}_{s}^{p}\left(\Delta_{\ell, j}\right)} .
$$

The first term of (12) is $\leq \beta^{-\ell}\|g\|_{s}\left|h-h_{0}\right|_{\mathcal{C}_{s}^{q}\left(E_{k}\right)}|\varphi|_{\mathcal{C}_{s}^{q}\left(E_{k}\right)}$ and similarly for the fourth term. We must show $\left|h-h_{0}\right|_{\mathcal{C}_{s}^{q}(\Delta)}$ has order $\beta^{k}$.

Clearly, $\left|h-h_{0}\right|_{\infty} \leq \operatorname{Lip}^{u}(h) \beta_{0}^{k}$ since the separation time in $E_{k}$ is $\geq k$. To estimate the Hölder norm, let $x, y \in \gamma^{s}$. Then

$$
\left|h(x)-h_{0}(x)-h(y)-h_{0}(y)\right| \leq 2 \operatorname{Lip}^{u}(h) \beta_{0}^{k},
$$

estimating the $x$ and $y$ differences separately. On the other hand, since $h \in \mathcal{C}_{s}^{p}(\Delta)$,

$$
\left|h(x)-h_{0}(x)-h(y)-h_{0}(y)\right| \leq 2 H_{s}^{p}(h) d_{s}(x, y)^{p} .
$$

The Hölder constant is bounded by the minimum of the two estimates $2 \operatorname{Lip}^{u}(h) \beta_{0}^{k} d_{s}(x, y)^{-q}$ and $2 H_{s}^{p}(\varphi) d_{s}(x, y)^{p-q}$. This minimum is largest when the two estimates are equal, i.e., when $\beta_{0}^{k}=d_{s}(x, y)^{p}$. Thus $H_{s}^{q}\left(h-h_{0}\right) \leq C \beta_{0}^{k(p-q) / p} \leq C \beta^{k}$ since $\beta$ was chosen $\geq \beta_{0}^{(p-q) / p}$.

Remark 3.4. Since $|\cdot|_{w} \leq\|\cdot\|_{s}$, there exists a natural embedding of $\mathcal{B}$ into $\mathcal{B}_{w}$. Moreover, Lemmas 3.2 and 3.3 imply that $\operatorname{Lip}^{u}(\Delta) \hookrightarrow \mathcal{B} \hookrightarrow \mathcal{B}_{w} \hookrightarrow \mathcal{C}_{s}^{p}(\Delta)^{\prime}$. In fact, the inclusions are injective up to modification of $h \in \operatorname{Lip}^{u}(\Delta)$ on sets of $m$-measure zero. This can be proven as in [GL, Proposition 4.1]. Accordingly, we will consider $\mathcal{B}$ as a subset of $\mathcal{B}_{w}$ and $\operatorname{Lip}^{u}(\Delta)$ as a subset of $\mathcal{B}$ by identifying $h \in \operatorname{Lip}^{u}(\Delta)$ with the measure $h m$.

\subsection{Compactness.}

LEMMA 3.5. On a fixed $E \in \mathcal{P}_{\ell, j}^{k_{0}}$, the unit ball of $\|\cdot\|$ is compactly embedded in $|\cdot|_{w}$.

Proof. Let $\varepsilon>0$ be fixed. We will show that there are finitely many $\psi_{i} \in \mathcal{C}_{s}^{p}(E)$ such that, for any $h \in \operatorname{Lip}^{u}(\Delta)$ and any $\varphi \in \mathcal{C}_{s}^{p}(E)$ with $|\varphi|_{\mathcal{C}_{s}^{p}(E)} \leq 1$, there exists an $i$ such that

$$
\beta_{0}^{\ell}\left|f_{E} h \varphi d m-f_{E} h \psi_{i} d m\right| \leq b^{-1}\|h\| \varepsilon
$$


Now let $\varphi \in \mathcal{C}_{s}^{p}(E)$ be such that $|\varphi|_{\mathcal{C}_{s}^{p}(E)} \leq 1$. For $k \geq k_{0}$, let $E_{i}^{k}$ denote the finitely many $k$-cylinders in $E$. For $\varphi \in \mathcal{C}_{s}^{r}(E)$ and $x \in E_{i}^{k}$, define

$$
\varphi_{k, i}(x)=m\left(E_{i}^{k}\right)^{-1} \int_{\gamma^{u}(x) \cap E_{i}^{k}} \varphi d m_{\gamma},
$$

and note that $\varphi_{k, i} \in \mathcal{C}_{s}^{r}\left(E_{i}^{k}\right)$ by Lemma 3.1. Let $\mathcal{H}_{E}^{r}(k, i)$ be the space of such averaged Hölder functions generated by $\varphi \in \mathcal{C}_{S}^{r}(E)$. Since $p>q$, it is a standard consequence of the Arzela-Ascoli theorem that the unit ball of $\mathcal{H}_{E}^{p}(k, i)$ is compactly embedded in $\mathcal{H}_{E}^{q}(k, i)$ for each $i \geq 0, k \geq k_{0}$. Thus we may choose finitely many functions $\left\{\psi_{k, i, n}\right\}_{n=1}^{N} \subset$ $\mathcal{H}_{E}^{p}(k, i)$ which form an $\varepsilon$-covering in the $\mathcal{C}_{s}^{q}$-norm of the unit ball of $\mathcal{C}_{s}^{p}\left(E_{i}^{k}\right)$.

Choose $k$ such that $\beta^{k}<\varepsilon$ and let $\varphi_{k}=\sum_{i} \varphi_{k, i} 1_{E_{i}^{k}}$. On each $E_{i}^{k}$, choose $1 \leq n \leq N$ such that $\left|\psi_{k, i, n}-\varphi_{k, i}\right|_{\mathcal{C}_{s}^{q}\left(E_{i}^{k}\right)} \leq \varepsilon$ and let $\psi_{k}=\sum_{i} \psi_{k, i, n} 1_{E_{i}^{k}}$. Then $\varphi_{k}, \psi_{k} \in \mathcal{C}_{s}^{p}(E)$ by Lemma 3.1 and $\left|\psi_{k}-\varphi_{k}\right|_{\mathcal{C}_{s}^{q}(E)} \leq \varepsilon$. Now,

$$
\left|\int_{E} h \varphi d m-\int_{E} h \psi_{k} d m\right| \leq\left|\int_{E} h\left(\varphi-\varphi_{k}\right) d m\right|+\left|\int_{E} h\left(\varphi_{k}-\psi_{k}\right) d m\right| .
$$

We estimate the first term of (13) using the strong unstable norm,

$$
\left|\int_{E} h\left(\varphi-\varphi_{k}\right) d m\right|=\sum_{i} m\left(E_{i}^{k}\right)\left|f_{E_{i}^{k}} h \varphi d m-f_{E_{i}^{k}} h \varphi_{k, i} d m\right| \leq m(E) \beta^{k-\ell}\|h\|_{u} .
$$

We estimate the second term of (13) using the strong stable norm,

$$
\left|\int_{E} h\left(\varphi_{k}-\psi_{k}\right) d m\right| \leq m(E) \beta^{-\ell}\|h\|_{s}\left|\varphi_{k}-\psi_{k}\right|_{\mathcal{C}_{s}^{q}(E)} \leq m(E) \beta^{-\ell} \varepsilon\|h\|_{s} .
$$

Then since $\beta_{0}<\beta$ and $\beta^{k}<\varepsilon$, we have

$$
\beta_{0}^{\ell}\left|f_{E} h \varphi d m-f_{E} h \psi_{k} d m\right| \leq \beta_{0}^{\ell} \beta^{k-\ell}\|h\|_{u}+\beta_{0}^{\ell} \beta^{-\ell} \varepsilon\|h\|_{s} \leq b^{-1}\|h\| \varepsilon .
$$

LEMMA 3.6. The unit ball of $\mathcal{B}$ is compactly embedded in $\mathcal{B}_{w}$.

Proof. In light of Lemma 3.5, it suffices to show that the weak norm of $h \in \mathcal{B}$ can be approximated by considering its norm on only finitely many $k$-cylinders $E \in \mathcal{P}_{\ell, j}^{k}$. This will follow from the fact that $\beta_{0}<\beta$ and the averaging property of the strong unstable norm.

Notice that for $h \in \operatorname{Lip}^{u}(\Delta), E \in \mathcal{P}_{\ell, j}^{k}$ and $\varphi \in \mathcal{C}_{s}^{p}(E)$,

$$
\left|\int_{E} h \varphi d m\right| \leq m(E) \beta^{-\ell}\|h\|_{s}|\varphi|_{\mathcal{C}_{s}^{q}(E)}
$$

so that $|h|_{w\left(\mathcal{P}_{\ell, j}^{k}\right)} \leq \beta_{0}^{\ell} \beta^{-\ell}\|h\|_{s}$.

Now fix $\varepsilon>0$ and choose $L$ large enough that $\left(\beta_{0} \beta^{-1}\right)^{L}<\varepsilon$. Then

$$
|h|_{w}=\sup _{\ell<L} \sup _{j, k \geq 0}|h|_{w\left(\mathcal{P}_{\ell, j}^{k}\right)}+\varepsilon\|h\|_{s} .
$$

Since there are only finitely many $\Delta_{\ell, j}$ per level $\ell$, it remains to show that the weak norm of $h$ on $k$-cylinders can be made small for large $k$. 
Choose $K$ so that $\beta^{K}<\varepsilon$. Then any cylinder set $E_{r} \in \mathcal{P}_{\ell, j}^{r}$ with $r \geq K$ is contained in some $K$-cylinder $E_{K}$. For $\varphi \in \mathcal{C}_{S}^{p}\left(E_{r}\right)$, let $\varphi_{E_{r}}$ be as in $\$ 2.2$ and let $\tilde{\varphi}_{E_{r}}$ denote the extension of $\varphi_{E_{r}}$ to $E_{K}$. Then

$$
\beta_{0}^{\ell}\left|f_{E_{r}} h \varphi d m-f_{E_{K}} h \tilde{\varphi}_{E_{r}} d m\right| \leq \beta_{0}^{\ell} \beta^{K-\ell}\|h\|_{u}|\varphi|_{\mathcal{C}_{s}^{p}\left(E_{r}\right)} .
$$

With this choice of $K$ and $L$ and letting $J_{\ell}$ denote the number of $\Delta_{\ell, j}$ on level $\ell$, we have

$$
|h|_{w}=\sup _{\ell<L} \sup _{j \leq J_{\ell}} \sup _{k \leq K}|h|_{w\left(\mathcal{P}_{\ell, j}^{k}\right)}+b^{-1}\|h\| \varepsilon,
$$

which implies the desired compactness.

\section{Lasota-Yorke-type estimates}

In this section we prove Proposition 2.3. By density of $\operatorname{Lip}^{u}(\Delta)$ in $\mathcal{B}$ and $\mathcal{B}_{w}$, it suffices to derive these inequalities for $h \in \operatorname{Lip}^{u}(\Delta)$ once we show $\mathcal{L}$ is continuous on $(\mathcal{B},\|\cdot\|)$. To avoid repeating estimates, we postpone the proof of this fact to $\S 4.4$.

4.1. Weak norm estimate. Let $h \in \operatorname{Lip}^{u}(\Delta)$ and $k \geq 0$ be fixed. Choose $E \in \mathcal{P}_{\ell, j}^{k}$ and $\varphi \in \mathcal{C}_{S}^{p}(E)$ with $|\varphi|_{\mathcal{C}_{s}^{p}(E)} \leq 1$.

Case 1. $n \leq \ell$. Notice that $E^{\prime}=F^{-n} E$ is an $n+k$ cylinder in $\Delta_{\ell-n, j^{\prime}}$, i.e., $E^{\prime} \in$ $\mathcal{P}_{\ell-n, j^{\prime}}^{k+n}$

$$
\int_{E} \mathcal{L}^{n} h \varphi d m=\int_{F^{-n} E} h \varphi \circ F^{n} d m \leq m\left(E^{\prime}\right) \beta_{0}^{n-\ell}|h|_{w\left(\mathcal{P}_{\ell-n, j^{\prime}}^{k+n}\right)}\left|\varphi \circ F^{n}\right|_{\mathcal{C}_{s}^{p}\left(E^{\prime}\right)} .
$$

Since $\left.J_{m} F^{n}\right|_{E^{\prime}} \equiv 1$ and $d_{s}\left(F^{n} x, F^{n} y\right)=\alpha^{n} d_{s}(x, y)$ for all $x, y \in E^{\prime}$, we have $m\left(E^{\prime}\right)=$ $m(E)$ and $\left|\varphi \circ F^{n}\right|_{\mathcal{C}_{s}^{p}\left(E^{\prime}\right)} \leq|\varphi|_{\mathcal{C}_{s}^{p}(E)}$. Putting this together with (14) and taking the supremum over $\varphi \in \mathcal{C}_{s}^{p}(E)$ and $E \in \mathcal{P}_{\ell, j}^{k}$, yields

$$
\left|\mathcal{L}^{n} h\right|_{w\left(\mathcal{P}_{\ell, j}^{k}\right)} \leq \beta_{0}^{n}|h|_{w\left(\mathcal{P}_{\ell-n, j^{\prime}}^{k+n}\right.} .
$$

Case 2. $n>\ell$. First consider the case $\ell=0$ and $E \in \mathcal{P}_{0, j}^{k}$. Note that $F^{-n} E$ comprises a countable union of $(n+k)$-cylinders, $F^{-n} E=\bigcup E^{\prime}, E^{\prime} \in \mathcal{P}_{\ell^{\prime}, j^{\prime}}^{n+k}$. Then by (14),

$$
\int_{E} \mathcal{L}^{n} h \varphi d m \leq \sum_{E^{\prime} \subset F^{-n} E} m\left(E^{\prime}\right) \beta_{0}^{-\ell^{\prime}}|h|_{w\left(\mathcal{P}_{\ell^{\prime}, j^{\prime}}^{k+n}\right.}\left|\varphi \circ F^{n}\right|_{\mathcal{C}_{s}^{p}\left(E^{\prime}\right)}
$$

where $E^{\prime} \subseteq \Delta_{\ell^{\prime}, j^{\prime}}$. To estimate $\left|\varphi \circ F^{n}\right|_{\mathcal{C}_{s}^{p}\left(E^{\prime}\right)}$, take $x, y \in \gamma^{s} \subset E^{\prime}$ and write

$$
\left|\varphi \circ F^{n}(x)-\varphi \circ F^{n}(y)\right| \leq H_{s}^{p}(\varphi) d_{s}\left(F^{n} x, F^{n} y\right)^{p} \leq H_{s}^{p}(\varphi) C_{0} \alpha^{p n} d_{s}(x, y)^{p}
$$

by (2). Since $\left|\varphi \circ F^{n}\right|_{\infty}=|\varphi|_{\infty}$, we conclude $\left|\varphi \circ F^{n}\right|_{\mathcal{C}_{s}^{p}\left(E^{\prime}\right)} \leq C_{0}|\varphi|_{\mathcal{C}_{s}^{p}(E)}$.

Due to bounded distortion given by Property (P4)(b) and Lemma 2.1, we have $m\left(E^{\prime}\right) / m(E) \leq C\left(m\left(E_{n}^{\prime}\right) / m\left(\Delta_{0, j}\right)\right)$ where $E_{n}^{\prime}$ is the $n$-cylinder containing $E^{\prime}$. Using the fact that there are only finitely many $\Delta_{0, j}$, we record the following estimate for future use. There exists $C>0$ such that,

$$
m\left(E^{\prime}\right) \leq C m\left(E_{n}^{\prime}\right) m(E) .
$$


Now (16) becomes,

$\int_{E} \mathcal{L}^{n} h \varphi d m \leq \sum_{E^{\prime} \subset F^{-n} E} C C_{0} \beta_{0}^{-\ell^{\prime}}|h|_{w} m\left(E_{n}^{\prime}\right) m(E) \leq C^{\prime}|h|_{w} m(E) \sum_{\ell^{\prime}, j^{\prime}} \beta_{0}^{-\ell^{\prime}} m\left(\Delta_{\ell^{\prime}, j^{\prime}}\right)$

where the sum is finite since $\theta<\beta_{0}$. Dividing by $m(E)$ and taking the supremum over $\varphi \in \mathcal{C}_{s}^{p}(E)$ and $E \in \mathcal{P}_{\ell, j}^{k}$, we have

$$
\left|\mathcal{L}^{n} h\right|_{w\left(\mathcal{P}_{0, j}^{k}\right)} \leq C^{\prime}|h|_{w} .
$$

Now for $n>\ell$, we use (15) and (19) to prove (6),

$$
\left|\mathcal{L}^{n} h\right|_{w\left(\mathcal{P}_{\ell, j}^{k}\right)} \leq \beta_{0}^{\ell}\left|\mathcal{L}^{n-\ell} h\right|_{w\left(\mathcal{P}_{0, j^{\prime}}^{k+\ell}\right)} \leq C^{\prime} \beta_{0}^{\ell}|h|_{w}
$$

4.2. Strong stable norm estimate. Fix $h \in \operatorname{Lip}^{u}(\Delta)$ and $k \geq 0$. Choose $E \in \mathcal{P}_{\ell, j}^{k}$ and $\varphi \in \mathcal{C}_{s}^{q}(E)$ with $|\varphi|_{\mathcal{C}_{s}^{q}(E)} \leq 1$

Case 1. $n \leq \ell$. Following (14) with $\mathcal{C}_{s}^{q}(\Delta)$ in place of $\mathcal{C}_{s}^{p}(\Delta)$, we obtain

$$
\left\|\mathcal{L}^{n} h\right\|_{s\left(\mathcal{P}_{\ell, j}^{k}\right)} \leq \beta^{n}\|h\|_{s\left(\mathcal{P}_{\ell-n, j^{\prime}}^{k+n}\right.} .
$$

Case 2. $n>\ell$. As in $\S 4.1$, we first let $\ell=0$ and fix $E \in \mathcal{P}_{0, j}^{k}$.

$$
\int_{E} \mathcal{L}^{n} h \varphi d m=\int_{F^{-n} E} h\left(\varphi \circ F^{n}-\bar{\varphi}\right) d m+\int_{F^{-n} E} h \bar{\varphi} d m
$$

where $\bar{\varphi}(x)=\int_{\gamma^{s}(x)} \varphi \circ F^{n} d \mu^{s}$ is constant on each $\gamma^{s} \subset F^{-n} E$. Denoting by $E^{\prime}$ the components of $F^{-n} E, E^{\prime} \in \mathcal{P}_{\ell^{\prime}, j^{\prime}}^{k+n}$, we estimate the first term of (21) by

$$
\int_{F^{-n} E} h\left(\varphi \circ F^{n}-\bar{\varphi}\right) d m \leq \sum_{E^{\prime} \subset F^{-n} E} \beta^{-\ell^{\prime}}\|h\|_{s}\left|\varphi \circ F^{n}-\bar{\varphi}\right|_{\mathcal{C}_{s}^{q}\left(E^{\prime}\right)} m\left(E^{\prime}\right) .
$$

Since $\varphi \circ F^{n}$ is continuous on $\gamma^{s} \in \Gamma^{s}$ and $\mu^{s}$ is a probability measure, we have $\varphi \circ$ $F^{n}\left(z_{1}\right) \leq \bar{\varphi} \leq \varphi \circ F^{n}\left(z_{2}\right)$ for some $z_{1}, z_{2} \in \gamma^{s}$. Thus using (17) we estimate

$$
\left|\varphi \circ F^{n}-\bar{\varphi}\right|_{\mathcal{C}_{s}^{q}\left(E^{\prime}\right)} \leq C \alpha^{q n}|\varphi|_{\mathcal{C}_{s}^{q}(E)} .
$$

Putting this together with (18) and (22), we obtain

$$
\int_{F^{-n} E} h\left(\varphi \circ F^{n}-\bar{\varphi}\right) d m \leq C \sum_{\ell^{\prime}, j^{\prime}} \beta^{-\ell^{\prime}} m\left(E_{n}^{\prime}\right) m(E)\|h\|_{s} \alpha^{q n} \leq C^{\prime} \alpha^{q n}\|h\|_{s} m(E) .
$$

To estimate the second term of (21), we note that $|\bar{\varphi}|_{\mathcal{C}_{s}^{p}\left(E^{\prime}\right)} \leq|\varphi|_{\infty} \leq 1$ since $\bar{\varphi}$ is constant along stable leaves. Then, again using (18) and the fact that $\theta<\beta_{0}$,

$$
\int_{F^{-n} E} h \bar{\varphi} d m \leq \sum_{E^{\prime} \subset F^{-n} E} m\left(E^{\prime}\right) \beta_{0}^{-\ell^{\prime}}|h|_{w}|\bar{\varphi}|_{\mathcal{C}_{s}^{p}\left(E^{\prime}\right)} \leq C|h|_{w} m(E) \sum_{\ell^{\prime}, j^{\prime}} \beta_{0}^{-\ell^{\prime}} m\left(\Delta_{\ell^{\prime}, j^{\prime}}\right) .
$$

Combining (23) and (24), dividing by $m(E)$ and taking the appropriate suprema yields

$$
\left\|\mathcal{L}^{n} h\right\|_{s\left(\mathcal{P}_{0, j}^{k}\right)} \leq C \alpha^{q n}\|h\|_{s}+C|h|_{w} .
$$

Now for $n>\ell$, we combine (20), (25) and the fact that $\alpha^{q}<\beta$ to obtain (7),

$$
\left\|\mathcal{L}^{n} h\right\|_{s\left(\mathcal{P}_{\ell, j}^{k}\right)} \leq \beta^{\ell}\left\|\mathcal{L}^{n-\ell} h\right\|_{s\left(\mathcal{P}_{0, j^{\prime}}^{k+\ell}\right)} \leq \beta^{\ell}\left(C \alpha^{q(n-\ell)}\|h\|_{s}+C|h|_{w}\right) .
$$


4.3. Strong unstable norm estimate. Fix $h \in \operatorname{Lip}^{u}(\Delta)$ and $E_{k} \in \mathcal{P}_{\ell, j}^{k}$. For $r \geq k$, let $E_{r} \in \mathcal{P}_{\ell, j}^{r}$ be such that $E_{r} \subseteq E_{k}$. For $\varphi \in \mathcal{C}_{s}^{p}\left(E_{r}\right)$, let

$$
\varphi_{E_{r}}(x)=m\left(E_{r}\right)^{-1} \int_{\gamma^{u}(x) \cap E_{r}} \varphi d m_{\gamma}
$$

be defined as in $\$ 2.2$ and let $\tilde{\varphi}_{E_{r}}(x)=\varphi_{E_{r}}\left(\gamma^{u}(x)\right)$ be the extension of $\varphi_{E_{r}}$ to $E_{k}$.

Case 1. $n \leq \ell$. Since $m\left(E_{r}\right)=m\left(F^{-n} E_{r}\right)$ and similarly for $E_{k}$, we have

$$
f_{E_{r}} \mathcal{L}^{n} h \varphi d m-f_{E_{k}} \mathcal{L}^{n} h \tilde{\varphi}_{E_{r}} d m=f_{F^{-n} E_{r}} h \varphi \circ F^{n} d m-f_{F^{-n} E_{k}} h \tilde{\varphi}_{E_{r}} \circ F^{n} d m .
$$

As before, $\left|\varphi \circ F^{n}\right|_{\mathcal{C}_{s}^{p}\left(F^{-n} E_{r}\right)} \leq|\varphi|_{\mathcal{C}_{s}^{p}\left(E_{r}\right)}$. Let $\left(\varphi \circ F^{n}\right)_{E_{r}^{n}}$ be the average of $\varphi \circ F^{n}$ on unstable leaves in the $(r+n)$-cylinder $E_{r}^{n}:=F^{-n} E_{r}$. Since $\left(\varphi \circ F^{n}\right)_{E_{r}^{n}}$ is constant on unstable leaves, we may extend it to $F^{-n} E_{k} \in \mathcal{P}_{\ell-n, j^{\prime}}^{k+n}$. It follows that $\tilde{\varphi}_{E_{r}} \circ F^{n}=$ $\left(\varphi \circ F^{n}\right)_{E_{r}^{n}}$ since $J_{\gamma} F^{n} \equiv 1$ on $F^{-n} E_{k}$.

$$
\left|f_{F^{-n} E_{r}} h \varphi \circ F^{n} d m-f_{F^{-n} E_{k}} h\left(\varphi \circ F^{n}\right)_{E_{r}^{n}} d m\right| \leq\|h\|_{u\left(\mathcal{P}_{\ell-n, j^{\prime}}^{k+n}\right)} \beta^{k+n-\ell}|\varphi|_{\mathcal{C}_{s}^{p}(E)} .
$$

Dividing by $\beta^{k-\ell}$ and taking the supremum over $\varphi \in \mathcal{C}_{s}^{p}\left(E_{r}\right)$ and $r \geq k$, we conclude that

$$
\left\|\mathcal{L}^{n} h\right\|_{u\left(\mathcal{P}_{\ell, j}^{k}\right)} \leq \beta^{n}\|h\|_{u\left(\mathcal{P}_{\ell-n, j^{\prime}}^{k+n}\right.} .
$$

Case 2. $n>\ell$. As before, we first consider the case $\ell=0$ and fix $E_{r} \subseteq E_{k} \subseteq \Delta_{0, j}$. Let $E_{k}^{n}$ denote an $(n+k)$-cylinder in $F^{-n} E_{k}$ and similarly for $E_{r}^{n} \subset E_{k}^{n}$. As usual, define $\left(\varphi \circ F^{n}\right) E_{r}^{n}$ to be the average of $\varphi \circ F^{n}$ on unstable leaves in $E_{r}^{n}$, extended to $E_{k}^{n}$. Now,

$$
\begin{aligned}
f_{E_{r}} & \mathcal{L}^{n} h \varphi d m-f_{E_{k}} \mathcal{L}^{n} h \tilde{\varphi}_{E_{r}} d m \\
= & \sum_{E_{k}^{n} \subset F^{-n} E_{k}} \frac{m\left(E_{r}^{n}\right)}{m\left(E_{r}\right)}\left[f_{E_{r}^{n}} h \varphi \circ F^{n} d m-f_{E_{k}^{n}} h\left(\varphi \circ F^{n}\right)_{E_{r}^{n}} d m\right] \\
& +\sum_{E_{k}^{n} \subset F^{-n} E_{k}}\left[\frac{m\left(E_{r}^{n}\right)}{m\left(E_{r}\right)}-\frac{m\left(E_{k}^{n}\right)}{m\left(E_{k}\right)}\right] f_{E_{k}^{n}} h\left(\varphi \circ F^{n}\right)_{E_{r}^{n}} d m \\
& +\sum_{E_{k}^{n} \subset F^{-n} E_{k}} \frac{m\left(E_{k}^{n}\right)}{m\left(E_{k}\right)} f_{E_{k}^{n}} h\left[\left(\varphi \circ F^{n}\right)_{E_{r}^{n}}-\tilde{\varphi}_{E_{r}} \circ F^{n}\right] d m .
\end{aligned}
$$

Label the three sums of (27) by (1), (2) and (3) respectively. To estimate (1), recall that, by (17), $\left|\varphi \circ F^{n}\right|_{\mathcal{C}_{s}^{p}\left(E_{r}^{n}\right)} \leq C_{0}|\varphi|_{\mathcal{C}_{s}^{p}\left(E_{r}\right)}$. Let $E^{n}$ denote the $n$-cylinder containing $E_{r}^{n}$ (and $\left.E_{k}^{n}\right)$ on level $\ell^{\prime}\left(E^{n}\right)$. We use (18) to estimate

$$
(1) \leq \sum_{E_{r}^{n} \subset F^{-n} E_{r}} C m\left(E^{n}\right) \beta^{n+k-\ell^{\prime}\left(E^{n}\right)}\|h\|_{u}\left|\varphi \circ F^{n}\right|_{\mathcal{C}_{s}^{p}\left(E_{r}^{n}\right)} \leq C^{\prime} \beta^{n+k}\|h\|_{u} .
$$

To estimate (2), note that $m(A)=m_{\gamma}(\gamma \cap A)$ for any $s$-subset $A$ by Lemma 2.1(1). By Lemma 2.1(3) and (18), there exist $x, y \in E_{k}^{n}$ such that

$$
\left|\frac{m\left(E_{r}^{n}\right)}{m\left(E_{r}\right)}-\frac{m\left(E_{k}^{n}\right)}{m\left(E_{k}\right)}\right| \leq\left|\frac{J_{\gamma} F^{n}(y)}{J_{\gamma} F^{n}(x)}-1\right| \frac{m\left(E_{k}^{n}\right)}{m\left(E_{k}\right)} \leq C \alpha^{k / 2} m\left(E^{n}\right)
$$


since $s(x, y) \geq n+k$. Recall that $\left|\left(\varphi \circ F^{n}\right)_{E_{r}^{n}}\right|_{\mathcal{C}_{s}^{q}\left(E_{r}^{n}\right)} \leq\left|\varphi \circ F^{n}\right|_{\mathcal{C}_{s}^{q}\left(E_{r}^{n}\right)} \leq C_{0}|\varphi|_{\mathcal{C}_{s}^{q}\left(E_{r}\right)}$ by Lemma 3.1 and (17). Using this estimate and (29),

$$
\text { (2) } \leq \sum_{E_{k}^{n} \subset F^{-n} E_{k}} C \alpha^{k / 2} m\left(E^{n}\right)\|h\|_{s} \beta^{-\ell^{\prime}} C_{0}|\varphi|_{\mathcal{C}_{s}^{q}\left(E_{r}\right)} \leq C^{\prime}\|h\|_{s} \alpha^{k / 2} .
$$

In order to estimate (3), we need the following preliminary lemma.

Lemma 4.1. For $E_{k}^{n} \subset \Delta_{\ell^{\prime}, j^{\prime}}$, let $\left(\varphi \circ F^{n}\right)_{E_{r}^{n}}$ and $\tilde{\varphi}_{E_{r}}$ be as above. There exists $C>0$ depending only on the distortion of $F$ such that

$$
\left|\left(\varphi \circ F^{n}\right)_{E_{r}^{n}}-\tilde{\varphi}_{E_{r}} \circ F^{n}\right|_{\mathcal{C}_{s}^{q}\left(E_{k}^{n}\right)} \leq C \alpha^{r(p-q) / 2 p}|\varphi|_{\mathcal{C}_{s}^{p}\left(E_{r}\right)} .
$$

Proof. For $x \in E_{k}^{n}$,

$$
\tilde{\varphi}_{E_{r}} \circ F^{n}(x)=m\left(E_{r}\right)^{-1} \int_{\gamma^{u}\left(F^{n} x\right) \cap E_{r}} \varphi d m_{\gamma}=m\left(E_{r}\right)^{-1} \int_{\gamma^{u}(x) \cap E_{r}^{n}} \varphi \circ F^{n} J_{\gamma} F^{n} d m_{\gamma} .
$$

Thus

$$
\left|\left(\varphi \circ F^{n}\right)_{E_{r}^{n}}(x)-\tilde{\varphi}_{E_{r}} \circ F^{n}(x)\right|=\frac{1}{m\left(E_{r}^{n}\right)}\left|\int_{\gamma^{u}(x) \cap E_{r}^{n}} \varphi \circ F^{n}\left(1-\frac{m\left(E_{r}^{n}\right) J_{\gamma} F^{n}}{m\left(E_{r}\right)}\right) d m_{\gamma}\right| .
$$

Now $m\left(E_{r}\right) / m\left(E_{r}^{n}\right)$ is the average value of $J_{\gamma} F^{n}$ on $\gamma^{u}(x) \cap E_{r}^{n}$ since $m\left(E_{r}^{n}\right)=$ $m_{\gamma}\left(\gamma^{u}(x) \cap E_{r}^{n}\right)$ and $m\left(E_{r}\right)=m_{\gamma}\left(\gamma^{u}\left(F^{n} x\right) \cap E_{r}\right)$ by Lemma 2.1(1). Lemma 2.1(3) implies

$$
\left|1-\frac{m\left(E_{r}^{n}\right) J_{\gamma} F^{n}}{m\left(E_{r}\right)}\right| \leq C_{1} \alpha^{r / 2}
$$

since the separation time for any two points in $E_{r}^{n}$ is at least $n+r$. Thus (31) becomes

$$
\left|\left(\varphi \circ F^{n}\right)_{E_{r}^{n}}(x)-\tilde{\varphi}_{E_{r}} \circ F^{n}(x)\right| \leq|\varphi|_{\infty} C_{1} \alpha^{r / 2} .
$$

It remains to estimate the Hölder constant of the difference along stable leaves. Let $y \in \gamma^{s}(x) \subset E_{k}^{n}$. On the one hand we have

$$
\left|\tilde{\varphi}_{E_{r}} \circ F^{n}(x)-\left(\varphi \circ F^{n}\right)_{E_{r}^{n}}(x)-\tilde{\varphi}_{E_{r}} \circ F^{n}(y)+\left(\varphi \circ F^{n}\right)_{E_{r}^{n}}(y)\right| \leq 2|\varphi|_{\infty} C_{1} \alpha^{r / 2},
$$

using (32) for the $x$ and $y$ differences separately. On the other hand, using (17), (2) and Lemma 3.1, we have

$$
\begin{aligned}
& \left|\tilde{\varphi}_{E_{r}} \circ F^{n}(x)-\left(\varphi \circ F^{n}\right)_{E_{r}^{n}}(x)-\tilde{\varphi}_{E_{r}} \circ F^{n}(y)+\left(\varphi \circ F^{n}\right)_{E_{r}^{n}}(y)\right| \\
& \quad \leq H^{p}\left(\left.\tilde{\varphi}_{E_{r}}\right|_{F^{n}\left(\gamma^{s}\right)}\right) d_{s}\left(F^{n} x, F^{n} y\right)^{p}+H^{p}\left(\left(\varphi \circ F^{n}\right) E_{r}^{n} \mid \gamma^{s}\right) d_{s}(x, y)^{p} \\
& \quad \leq H_{s}^{p}(\varphi) C_{0} \alpha^{p n} d_{s}(x, y)^{p}+H_{s}^{p}\left(\varphi \circ F^{n}\right) d_{s}(x, y)^{p} \leq 2 C_{0} H_{s}^{p}(\varphi) \alpha^{p n} d_{s}(x, y)^{p} .
\end{aligned}
$$

The Hölder constant is bounded by the minimum of the two estimates, $2 C_{1} \alpha^{r / 2} d_{s}(x, y)^{-q}$ and $2 C_{0} \alpha^{p n} d_{s}(x, y)^{p-q}$. This minimum is largest when the two quantities are equal, i.e., when $d_{s}(x, y)^{p}=\alpha^{-n p} \alpha^{r / 2} C_{1} / C_{0}$. Thus

$$
H_{s}^{q}\left(\tilde{\varphi}_{r} \circ F^{n}-\left(\varphi \circ F^{n}\right)_{n+r}\right) \leq C H_{s}^{p}(\varphi) \alpha^{q n} \alpha^{r(p-q) / 2 p}
$$

which, together with (32), completes the proof of the lemma. 
We are now ready to estimate (3) using the strong stable norm.

$$
\text { (3) } \leq \sum_{\ell^{\prime}, j^{\prime}} C\|h\|_{s} \beta^{-\ell^{\prime}} m\left(\Delta_{\ell^{\prime}, j^{\prime}}\right)\left|\left(\varphi \circ F^{n}\right)_{E_{r}^{n}}-\tilde{\varphi}_{E_{r}} \circ F^{n}\right|_{\mathcal{C}_{s}^{q}\left(E_{k}^{n}\right)} \leq C\|h\|_{s} \alpha^{r(p-q) / 2 p}
$$

by Lemma 4.1. Combining (28), (30) and (33) in (27), using the fact that $\alpha^{(p-q) / 2 p} \leq \beta$, and taking the appropriate suprema, we have

$$
\left\|\mathcal{L}^{n} h\right\|_{u\left(\mathcal{P}_{0, j}^{k}\right)} \leq C \beta^{n}\|h\|_{u}+C\|h\|_{s} .
$$

Now for $n>\ell$, we combine (26) and (34) to prove (8),

$$
\left\|\mathcal{L}^{n} h\right\|_{u\left(\mathcal{P}_{\ell, j}^{k}\right)} \leq \beta^{\ell}\left\|\mathcal{L}^{n-\ell} h\right\|_{u\left(\mathcal{P}_{0, j^{\prime}}^{k+\ell}\right)} \leq \beta^{\ell}\left(C \beta^{n-\ell}\|h\|_{u}+C\|h\|_{s}\right)
$$

4.4. Continuity of $\mathcal{L}$. Since $\mathcal{L}$ is linear, it suffices to show $\mathcal{L}$ is bounded on $\mathcal{B}$. Note that for $h \in \mathcal{B}$, we can express the strong stable norm given by (4) as

$$
\|h\|_{s\left(\mathcal{P}_{\ell, j}^{k}\right)}=\beta^{\ell} \sup _{E \in \mathcal{P}_{\ell, j}^{k}|\varphi|_{\mathcal{C}_{s}^{q}(E)} \leq 1} m(E)^{-1} h\left(\varphi \chi_{E}\right)
$$

where $\chi_{E}$ is the indicator function of the set $E$. Similarly, defining $\varphi_{E_{r}}$ and $\tilde{\varphi}_{E_{r}}$ as in $\S 2.2$, we can express the strong unstable norm given by (5) as

$$
\|h\|_{u\left(\mathcal{P}_{\ell, j}^{k}\right)}=\sup _{E_{k} \in \mathcal{P}_{\ell, j}^{k}} \sup _{E_{r} \subset E_{k}|\varphi|_{\mathcal{C}_{S}^{p}\left(E_{r}\right)} \leq 1} \sup \beta^{\ell-k}\left|m\left(E_{r}\right)^{-1} h\left(\varphi \chi_{E_{r}}\right)-m\left(E_{k}\right)^{-1} h\left(\tilde{\varphi}_{E_{r}} \chi_{E_{k}}\right)\right| .
$$

Now following the estimates of $\S 4.1$, we have for $E \in \mathcal{P}_{\ell, j}^{k}$ and $\varphi \in \mathcal{C}_{s}^{q}(E)$,

$$
\mathcal{L} h\left(\varphi \chi_{E}\right)=\sum_{E^{\prime} \subset F^{-1} E} h\left(\varphi \circ F \cdot \chi_{E^{\prime}}\right) \leq \sum_{E^{\prime} \subset F^{-1} E} m\left(E^{\prime}\right) \beta^{-\ell^{\prime}\left(E^{\prime}\right)}\|h\|_{s}|\varphi \circ F|_{\mathcal{C}_{s}^{q}\left(E^{\prime}\right)} .
$$

By (17), $|\varphi \circ F|_{\mathcal{C}_{s}^{q}\left(E^{\prime}\right)} \leq C_{0}|\varphi|_{\mathcal{C}_{s}^{q}(E)}$. Now if $\ell>0$, then there is only one $E^{\prime}=F^{-1} E$, $\ell^{\prime}\left(E^{\prime}\right)=\ell-1$, and $m\left(E^{\prime}\right)=m(E)$ so we conclude that

$$
\|\mathcal{L} h\|_{s\left(\mathcal{P}_{\ell, j}^{k}\right)} \leq C_{0} \beta\|h\|_{s} .
$$

On the other hand, if $\ell=0$, then by $(18), m\left(E^{\prime}\right) \leq C m\left(E_{1}^{\prime}\right) m(E)$ where $E_{1}^{\prime}$ is the 1-cylinder containing $E^{\prime}$. So (37) becomes

$$
\mathcal{L} h\left(\varphi \chi_{E}\right) \leq C C_{0} m(E)\|h\|_{s} \sum_{E^{\prime} \subset F^{-1} E} \beta^{-\ell^{\prime}} m\left(\Delta_{\ell^{\prime}, j^{\prime}}\right)
$$

and the sum is finite since $\beta>\theta$. Thus $\|\mathcal{L} h\|_{s} \leq C^{\prime}\|h\|_{s}$ as required.

Similarly, using (36) and following the estimates of $\S 4.3$, one sees that $\|\mathcal{L} h\|_{u} \leq$ $C\left(\|h\|_{s}+\|h\|_{u}\right)$.

\section{Spectral picture}

Proposition 2.3 and Lemma 3.6 imply that $\mathcal{L}: \mathcal{B} \circlearrowleft$ is quasi-compact with essential spectral radius bounded by $\beta$. In this section we study the peripheral spectrum of $\mathcal{L}$ on $\mathcal{B}$ and prove Theorems 1 and 2 . 


\subsection{Proof of Theorem 1 .}

LEMMA 5.1. The spectral radius of $\mathcal{L}$ on $\mathcal{B}$ is 1 . Its peripheral spectrum comprises measures and contains no Jordan blocks.

Proof. It is a direct consequence of (6) that the spectral radius of $\mathcal{L}$ is at most one. If it were strictly less than one, Lemma 3.2 would yield the contradiction

$$
|m(1)|=\left|\mathcal{L}^{n} m(1)\right|=\lim _{n \rightarrow \infty}\left|\mathcal{L}^{n} m(1)\right| \leq \lim _{n \rightarrow \infty} C\left\|\mathcal{L}^{n} m\right\|=0 .
$$

Now let $z$ be in the spectrum of $\mathcal{L}$ with $|z|=1$. Suppose there exist $h_{0}, h_{1} \in \mathcal{B}, h_{0} \neq 0$ such that $\mathcal{L} h_{0}=z h_{0}$ and $\mathcal{L} h_{1}=z h_{1}+h_{0}$. Then $\mathcal{L}^{n} h_{1}=z^{n} h_{1}+n z^{n-1} h_{0}$. Now (17) implies that $H_{s}^{p}\left(\varphi \circ F^{n}\right) \leq C_{0} \alpha^{p n} H_{s}^{p}(\varphi)$ for $\varphi \in \mathcal{C}_{s}^{p}(\Delta)$. So

$n\left|h_{0}(\varphi)\right| \leq\left|h_{1}(\varphi)\right|+\left|\mathcal{L}^{n} h_{1}(\varphi)\right|=\left|h_{1}(\varphi)\right|+\left|h_{1}\left(\varphi \circ F^{n}\right)\right| \leq 2 C\left|h_{1}\right|_{w}\left(|\varphi|_{\infty}+C_{0} H_{S}^{p}(\varphi)\right)$

for each $n$ by Lemma 3.2. Dividing by $n$ and taking the limit as $n \rightarrow \infty$ implies that $h_{0} \equiv 0$, contrary to our assumption.

It remains to show that the peripheral spectrum is comprised of measures. Suppose $h \in \mathcal{B}$ satisfies $\mathcal{L} h=z h$ for some $|z|=1$. By Lemma 3.2 and (17),

$$
|h(\varphi)|=\left|\mathcal{L}^{n} h(\varphi)\right|=\left|h\left(\varphi \circ F^{n}\right)\right| \leq C|h|_{w}\left(|\varphi|_{\infty}+C_{0} \alpha^{p n} H_{s}^{p}(\varphi)\right) .
$$

Letting $n \rightarrow \infty$ yields $|h(\varphi)| \leq C|h|_{w}|\varphi|_{\infty}$ for all $\varphi \in \mathcal{C}_{s}^{p}(\Delta)$, which implies $h$ is a measure.

Let $V_{\phi}$ denote the eigenspace in $\mathcal{B}$ corresponding to the eigenvalue $e^{i \phi}$. The absence of Jordan blocks in the peripheral spectrum implies that the spectral projectors $\Pi_{\phi}: \mathcal{B} \rightarrow V_{\phi}$ are well-defined and satisfy

$$
\Pi_{\phi} h=\lim _{n \rightarrow \infty} \frac{1}{n} \sum_{k=0}^{n-1} e^{-i k \phi} \mathcal{L}^{k} h
$$

where convergence is in the $\|\cdot\|$-norm so the limit holds with $h$ applied to any $\varphi \in \mathcal{C}_{S}^{p}(\Delta)$. By density, $\Pi_{\phi} \operatorname{Lip}^{u}(\Delta)=V_{\phi}$ so that for each $\eta \in V_{\phi}$ there is an $h \in \operatorname{Lip}^{u}(\Delta)$ such that $\Pi_{\phi} h=\eta$.

LeMma 5.2. Let $\eta \in V_{\phi}$. Then $\eta$ is absolutely continuous with respect to $\bar{v}$ where $\bar{v}:=\Pi_{0} 1$. Moreover, $\eta$ has absolutely continuous conditional measures with respect to Riemannian volume on unstable curves $\gamma \in \Gamma^{u}(\Delta)$ and $\eta \in \mathcal{G}$.

Proof. Let $h_{\eta} \in \operatorname{Lip}^{u}(\Delta)$ be such that $\Pi_{\phi} h_{\eta}=\eta$. Then for $\varphi \in \mathcal{C}_{s}^{p}(\Delta)$,

$$
|\eta(\varphi)| \leq \lim _{n \rightarrow \infty} \frac{1}{n} \sum_{k=0}^{n-1}\left|e^{-i k \phi} \mathcal{L}^{k} h_{\eta}(\varphi)\right| \leq \lim _{n \rightarrow \infty}\left|h_{\eta}\right|_{\infty} \frac{1}{n} \sum_{k=0}^{n-1} \mathcal{L}^{k} 1(|\varphi|)=\left|h_{\eta}\right|_{\infty} \overline{\mathcal{v}}(|\varphi|) .
$$

So $\eta=\psi \bar{v}$ for some $\psi$ with $|\psi|_{L^{\infty}(\bar{v})} \leq\left|h_{\eta}\right|_{\infty}$.

Since $\eta$ can be written as the limit of $\eta_{n}=(1 / n) \sum_{k=0}^{n-1} e^{-i k \phi} \mathcal{L}^{k} h_{\eta}$ and the conditional measures of $\eta_{n}$ on unstable leaves in $\Gamma^{u}$ have uniformly bounded and Lipschitz densities, the Markov structure of $\Delta$ and the regularity of $J^{u} F$ guarantee that this property passes to $\eta$ (this can be proved as in $[\mathbf{Y 1}, \S 2]$ ). 
LEMMA 5.3. The peripheral spectrum of $\mathcal{L}$ on $\mathcal{B}$ forms a group on the unit circle.

Proof. Let $\mathcal{U}_{F} \varphi=\varphi \circ F$ define the composition operator of $F$ on $L^{2}(\bar{v})$ and let $\mathcal{L}_{\bar{v}}$ be its dual, i.e., the transfer operator of $F$ with respect to $\bar{v}$. It is a standard fact of ergodic theory that the eigenvalues of $\mathcal{U}_{F}$ form a subgroup on the unit circle [W, §3.1]. It is then straightforward to show that the the peripheral eigenvalues of $\mathcal{L}_{\bar{v}}$ equal those of $\mathcal{U}_{F}$. In what follows, we will show that the eigenvalues on the unit circle of $\mathcal{L}_{\bar{v}}$ acting on $L^{2}(\bar{v})$ are precisely the peripheral spectrum of $\mathcal{L}$ on $\mathcal{B}$. We denote these sets by $\varrho\left(\mathcal{L}_{\bar{v}}\right)$ and $\varrho(\mathcal{L})$ respectively.

Suppose $\eta \in V_{\phi}$ and let $\psi \in L^{\infty}(\bar{v})$ satisfy $\eta=\psi \bar{v}$ by Lemma 5.2. For $\varphi \in \mathcal{C}_{b}^{0}(\Delta)$,

$$
e^{i \phi} \int \varphi \psi d \bar{\nu}=e^{i \phi} \eta(\varphi)=\mathcal{L} \eta(\varphi)=\eta(\varphi \circ F)=\int \varphi \circ F \cdot \psi d \bar{\nu}=\int \varphi \cdot \mathcal{L}_{\bar{v}} \psi d \bar{\nu}
$$

so that $\mathcal{L}_{\bar{v}} \psi=e^{i \phi} \psi$. Since $\psi \in L^{\infty}(\bar{v}) \subset L^{2}(\bar{v})$, we conclude that $\varrho(\mathcal{L}) \subset \varrho\left(\mathcal{L}_{\bar{v}}\right)$.

To show inclusion in the other direction, suppose $\psi \in L^{2}(\bar{v})$ satisfies $\mathcal{L}_{\bar{v}} \psi=e^{i \phi} \psi$. For $j \geq 0$, choose $h_{j} \in \operatorname{Lip}^{u}(\Delta) \cap \mathcal{C}_{s}^{p}(\Delta)$ such that $\left|\psi-h_{j}\right|_{L^{1}(\bar{v})} \leq 1 / j$. By Lemma 3.3(ii), $h_{j} \bar{v} \in \mathcal{B}$. Thus,

$$
\Pi_{\phi}\left(h_{j} \bar{v}\right)=\lim _{n \rightarrow \infty} \frac{1}{n} \sum_{k=0}^{n-1} e^{-i k \phi} \mathcal{L}^{k}\left(h_{j} \bar{v}\right)=: \eta_{j}=: \psi_{j} \bar{v} \in V_{\phi},
$$

by (38) and Lemma 5.2. On the other hand, for $\varphi \in \mathcal{C}_{b}^{0}(\Delta)$,

$$
\frac{1}{n} \sum_{k=0}^{n-1} e^{-i k \phi} \mathcal{L}^{k}\left(h_{j} \bar{v}\right)(\varphi)=\frac{1}{n} \sum_{k=0}^{n-1} e^{-i k \phi} \int \varphi \circ F^{k} \cdot\left(h_{j}-\psi\right) d \bar{v}+\int \varphi \psi d \bar{\nu} .
$$

The first term in (40) is bounded by

$$
\frac{1}{n} \sum_{k=0}^{n-1}|\varphi|_{\infty}\left|\psi-h_{j}\right|_{L^{1}(\bar{v})} \leq|\varphi|_{\infty} / j
$$

Combining (39) and (40), we have $\left|\int \varphi \psi d \bar{\nu}-\int \varphi \psi_{j} d \bar{\nu}\right| \leq|\varphi|_{\infty} / j$ for all $\varphi \in \mathcal{C}_{b}^{0}(\Delta)$ and each $j$. Since $V_{\phi}$ is finite dimensional and $\psi \bar{v}$ is approximated by elements of $V_{\phi}$, it must be that $\psi \bar{v} \in V_{\phi}$. Thus $\varrho\left(\mathcal{L}_{\bar{v}}\right) \subset \varrho(\mathcal{L})$.

We call $\Delta_{0, j}$ a recurrent base if $\mu$-almost every $x \in \Delta_{0, j}$ satisfies: $F^{n}(x) \in \Delta_{0, j}$ for infinitely many $n>0$. We call $\Delta_{0, j}$ transient if it is not recurrent.

Lemma 5.4. Let $\Delta^{(j)}$ be the tower above a recurrent base $\Delta_{0, j}$. Then the full Lebesgue measure of $\Delta^{(j)}$ belongs to a single ergodic component.

Proof. Choose $v \in V_{0}$ ergodic and fix a density point $x_{0}$ which is not on the boundary of any cylinder $E \in \mathcal{P}_{\ell, j}^{k}, k \geq 0$. Without loss of generality, take $x_{0} \in \Delta_{0, j}$, a recurrent base. Let $\gamma_{0}$ be the unstable leaf containing $x_{0}$ and let $\rho_{0}$ be the conditional density of $v$ on $\gamma_{0}$ which is Lipschitz by Lemma 5.2. Thus $\rho_{0}>0$ on an open subset $U$ in $\gamma_{0}$ containing $x_{0}$. Since $\Delta_{0, j}$ is recurrent, there exists $E \in \mathcal{P}_{1, j}^{k}$ with $E \cap \gamma_{0} \subset U$ such that $F^{n}(E)$ is a $u$-subset of $\Delta_{0, j}$ for some $n>0$. Thus the density of $\left.F_{*}^{n} \nu\right|_{F^{n}\left(E \cap \gamma_{0}\right)}$ is strictly positive. Since each stable leaf $\gamma^{s} \in \Gamma^{s}(\Delta)$ belongs to a single ergodic component, the full Lebesgue 
measure of $\Delta_{0, j}$ must belong to a single ergodic component. Thus the ergodic component of $v$ includes the full measure of each recurrent base (and hence the entire tower above each such base) which contains a density point of $v$.

Proof of Theorem 1. Lemma 5.4 allows us to assign each of the recurrent bases to a single ergodic component: two bases $\Delta_{0, j}$ and $\Delta_{0, j^{\prime}}$ are in the same ergodic component if and only if there exists $n, n^{\prime}$ such that $F^{n}\left(\Delta_{0, j}\right) \cap \Delta_{0, j^{\prime}} \neq \emptyset$ and $F^{n^{\prime}}\left(\Delta_{0, j^{\prime}}\right) \cap \Delta_{0, j} \neq \emptyset$.

Step 1. Mixing case. By transitivity the full Lebesgue measure of each base belongs to a single ergodic component by Lemma 5.4; therefore there can be only one invariant probability measure $v \in V_{0}$. Now suppose there exists $\eta \in V_{\phi}$ where $\phi=2 \pi p / q$ for some $p, q \in \mathbb{Z}^{+}$. Thus $v$ and $\eta$ are both invariant densities for $\mathcal{L}^{q}$. Since $F$ is mixing, $F^{q}$ is also transitive on $\Delta$ so $\mathcal{L}^{q}$ can have at most one invariant probability measure. Thus $\eta=v$. This proves item (i) of the theorem.

Step 2. Non-mixing case. First assume $F$ is transitive and periodic with period $p$. Then $\Delta$ decomposes under $F^{p}$ into $p$ transitive components, on each of which $F^{p}$ is mixing. By Step 1,1 is an eigenvalue of $\mathcal{L}^{p}$ with multiplicity $p$ and there are no other eigenvalues on the unit circle. The corresponding eigenvalues for $\mathcal{L}$ lie at the $p$ th roots of unity and it follows from transitivity that all the $p$ th roots are realized as simple eigenvalues. This proves (ii).

If $F$ is not transitive, we simply restrict to a single transitive component and apply (ii). Since quasi-compactness implies there are only finitely many components, (iii) follows.

\subsection{Proof of Theorem 2.}

(i) Theorem 2(i) is proven by Lemma 5.2.

(ii) Let $v$ be a physical measure. There exists $B_{v}$ with $\mu\left(B_{v}\right)>0$ such that for every $\varphi \in \mathcal{C}_{b}^{0}$,

$$
\lim _{n \rightarrow \infty} \frac{1}{n} \sum_{i=0}^{n-1} \varphi\left(F^{i} x\right)=v(\varphi) \quad \text { for all } x \in B_{v} .
$$

Let $x_{0} \in \Delta_{\ell, j}$ be a density point of $B_{\nu}$. Given $\varepsilon>0$, let $h_{\varepsilon} \in \operatorname{Lip}^{u}(\Delta)$ be a probability density with respect to $m$ supported on $\Delta_{\ell, j}$ such that $h_{\varepsilon}\left(B_{v}\right) \geq 1-\varepsilon$. For $\varphi \in C_{b}^{0}(\Delta)$, choose a set $B_{v, \varepsilon} \subset B_{v}$ on which (41) converges uniformly and $h_{\varepsilon}\left(B_{v} \backslash B_{v, \varepsilon}\right) \geq 1-2 \varepsilon$. Then

$$
\begin{aligned}
\Pi_{0} h_{\varepsilon}(\varphi) & =\lim _{n \rightarrow \infty} \frac{1}{n} \sum_{i=0}^{n-1} h_{\varepsilon}\left(\varphi \circ F^{i}\right) \\
& =\lim _{n \rightarrow \infty} \frac{1}{n} \sum_{i=0}^{n-1} h_{\varepsilon}\left(\varphi \circ F^{i} \cdot 1_{B_{v, \varepsilon}}\right)+h_{\varepsilon}\left(\varphi \circ F^{i} \cdot 1_{\Delta_{\ell, j} \backslash B_{v, \varepsilon}}\right) \\
& =h_{\varepsilon}\left(1_{B_{v, \varepsilon}}\right) v(\varphi)+\mathcal{O}\left(|\varphi|_{\infty} \varepsilon\right)=v(\varphi)+\mathcal{O}\left(|\varphi|_{\infty} \varepsilon\right) .
\end{aligned}
$$

Since $\Pi_{0} h_{\varepsilon} \in V_{0}$, this means $v$ can be approximated by elements of $V_{0}$ and so $v \in V_{0}$. The fact that $v$ is ergodic follows from its definition as a physical measure. 
Next we show that all ergodic elements of $V_{0}$ are physical measures. By Lemma 5.4, if $v \in V_{0}$ is an ergodic measure, its support is contained in a finite set of towers $\Delta^{(j)}$ above recurrent bases $\Delta_{0, j}$ and no other ergodic measure in $V_{0}$ can be supported on this set. Denote this set by $E_{v}$ and note that $v\left(E_{v}\right)=1$. Thus (41) converges to $v(\varphi)$ for $v$ almost every $x \in E_{v}$. Since the ergodic average is constant along stable leaves and $v$ has absolutely continuous conditional measures on $\gamma \in \Gamma^{u}(\Delta)$, we must have (41) converging for $\mu_{\gamma}$-almost every $x \in \gamma$ on every $\gamma \in \Gamma^{u}\left(\Delta_{\ell, j}\right), \Delta_{\ell, j} \subset E_{\nu}$.

On the other hand, if $\Delta_{0, j}$ is transient, every point in $\Delta^{(j)}$ belongs to a cylinder set of finite length $n$ which maps to a recurrent base $\Delta_{0, k}$ at time $n$. Let $\mathcal{C}_{k}$ denote the collection of such cylinder sets in $\Delta^{(j)}$ which map to the recurrent base $\Delta_{0, k}$. It is clear that $\mathcal{C}_{k}$ belongs to the basin of attraction of the unique element of $V_{0}$ supported on $\Delta_{0, k}$ and that the forward ergodic averages beginning in $\mathcal{C}_{k}$ converge to the same constant.

Thus to each ergodic element $v \in V_{0}$, we associate a basin $B_{v}$ comprising a maximal set of recurrent towers $E_{v}$ plus a collection of cylinder sets from transient towers. Since (41) converges for $\mu$-almost every $x \in B_{v}$ and $v\left(B_{v}\right)=v\left(E_{v}\right)=1, v$ is necessarily a physical measure.

It remains to show that the ergodic decomposition with respect to $\bar{v}$ corresponds to that with respect to $m$. Let $v$ be an ergodic element of $V_{0}$. Since $F^{-1} B_{v}=B_{v}$ and $B_{v}$ is a union of cylinder sets, $1_{B_{v}} \in \mathcal{C}_{S}^{p}(\Delta)$ and $1_{B_{v}} \circ F=1_{B_{v}}$, so

$$
\bar{v}\left(B_{v}\right)=\lim _{n \rightarrow \infty} \frac{1}{n} \sum_{i=0}^{n-1} \mathcal{L}^{i} m\left(B_{v}\right)=\lim _{n \rightarrow \infty} \frac{1}{n} \sum_{i=0}^{n-1} m\left(B_{v}\right)=m\left(B_{v}\right) .
$$

(iii) This is implied by the argument in (ii).

(iv) This is a standard corollary of the existence of a spectral gap given by Theorem 1(i). To obtain the slightly stronger result for $\psi \in \operatorname{Lip}^{u}(\Delta)$ and $\varphi \in \mathcal{C}_{s}^{p}(\Delta)$, note that $\psi \bar{v} \in \mathcal{G}$ and thus $\psi \bar{\nu} \in \mathcal{B}$ by Lemmas 5.2 and 3.3(i). Thus there exists $\sigma<1$ such that

$$
\int \psi \varphi \circ F^{n} d \bar{\nu}=\mathcal{L}^{n}(\psi \bar{\nu})(\varphi)=\bar{\nu}(\psi) \bar{\nu}(\varphi)+\mathcal{O}\left(\sigma^{n}\|\psi \bar{\nu}\|_{\mathcal{B}}|\varphi|_{\mathcal{C}_{s}^{p}(\Delta)}\right) .
$$

\section{Large-deviation estimates}

We connect the moment generating function $q(z)$ defined in $\$ 2.3 .1$ to the spectral properties of a generalized transfer operator as follows.

For $g \in \operatorname{Lip}^{u}(\Delta) \cap \mathcal{C}_{s}^{p}(\Delta)$, define the generalized transfer operator for $h \in \mathcal{B}$ by

$$
\mathcal{L}_{g} h(\varphi)=h\left(\varphi \circ F \cdot e^{g}\right) \quad \text { for all } \varphi \in \mathcal{C}_{s}^{p}(\Delta) .
$$

It is then a simple calculation that $\mathcal{L}_{g}^{n} h(\varphi)=h\left(\varphi \circ F^{n} \cdot e^{S_{n} g}\right)$. Now fix $g$ and for $z \in \mathbb{C}$ suppose that $\mathcal{L}_{z}=\mathcal{L}_{z g}$ is quasi-compact with a simple eigenvalue $\lambda_{z}$ of maximum modulus. Then, for $h \in \mathcal{B}$ such that $\Pi_{\lambda_{z}} h(1) \neq 0$,

$$
q(z)=\lim _{n \rightarrow \infty} \frac{1}{n} \log h\left(e^{z S_{n} g}\right)=\lim _{n \rightarrow \infty} \frac{1}{n} \log \mathcal{L}_{z}^{n} h(1)=\log \lambda_{z} .
$$

So $q(z)$ is well-defined if $\mathcal{L}_{z}$ has a spectral decomposition similar to that for $\mathcal{L}_{0}=\mathcal{L}$ given by Theorem 1 . With this in mind, we prove in $\S 6.2$. 
Proposition 6.1. For $g \in \operatorname{Lip}^{u}(\Delta) \cap \mathcal{C}_{s}^{p}(\Delta)$, let $g_{0}:=|g|_{\infty}<\infty$. Then, for $h \in \mathcal{B}$ and $n \geq 0$,

$$
\begin{aligned}
\left|\mathcal{L}_{g}^{n} h\right|_{w} & \leq C e^{n g_{0}}|g|_{\mathcal{C}_{s}^{p}(\Delta)}|h|_{w}, \\
\left\|\mathcal{L}_{g}^{n} h\right\|_{s} & \leq C e^{n g_{0}}|g|_{\mathcal{C}_{s}^{p}(\Delta)}\left(\beta^{n}\|h\|_{s}+|h|_{w}\right), \\
\left\|\mathcal{L}_{g}^{n} h\right\|_{u} & \leq C e^{n g_{0}}\left(\beta^{n}\|h\|_{u}|g|_{\mathcal{C}_{s}^{p}(\Delta)}+\|h\|_{s}\left(\operatorname{Lip}^{u}(g)+|g|_{\mathcal{C}_{s}^{q}(\Delta)}\right)\right) .
\end{aligned}
$$

This implies a Lasota-Yorke type inequality for $\mathcal{L}_{z}$ if $|z|<(-\ln \beta) / g_{0}$. In order to ensure that the spectral gap for $\mathcal{L}_{z}$ persists for small $|z|$, we introduce the following norm for operators from $\mathcal{B}$ to $\mathcal{B}_{w}$ :

$$
\left\|\mathcal{L}_{z}\right\| \mid=\sup \left\{\left|\mathcal{L}_{z} h\right|_{w}: h \in \mathcal{B},\|h\| \leq 1\right\} .
$$

In $\S 6.3$, we prove the following.

Lemma 6.2. There exists $C>0$, independent of $z \in \mathbb{C}$ and $g \in \operatorname{Lip}^{u}(\Delta) \cap \mathcal{C}_{s}^{p}(\Delta)$, such that

$$
\left\|\left|\mathcal{L}_{z}-\mathcal{L}_{0} \|\right| \leq C e^{|z| g_{0}}|z||g|_{\mathcal{C}_{s}^{q}(\Delta)} .\right.
$$

With this result, the perturbation results of [KL] imply that for small $|z|$, both the spectra and the spectral projectors of $\mathcal{L}_{z}$ vary continuously with $z$ so that if $\mathcal{L}=\mathcal{L}_{0}$ has a spectral gap, this gap persists for all $z \in \mathbb{C}$ with $|z|$ sufficiently small. Moreover, the eigenvalue of maximum modulus, $\lambda_{z}$, is real whenever $z$ is real.

6.1. Proof of Theorems 3 and 4. Proof of Theorem 3. We follow [RY] in our proof of Theorem 3, modifying the proof as necessary to generalize to non-invariant measures $\eta$.

Throughout this section, we assume $F$ is mixing and fix $g \in \mathcal{C}_{s}^{p}(\Delta) \cap \operatorname{Lip}^{u}(\Delta)$. We assume $|z|$ is sufficiently small so that $\mathcal{L}_{z}=\mathcal{L}_{z g}$ has a simple eigenvalue of maximum modulus $\lambda_{z}$, with corresponding eigenvector $v_{z} \in \mathcal{B}$.

Sublemma 6.3. The map $z \rightarrow \mathcal{L}_{z}$ is analytic for all $z \in \mathbb{C}$. Consequently, if $F$ is mixing, the map $z \rightarrow \lambda_{z}$ is analytic for $z$ in a complex neighborhood of 0 .

Proof. This follows directly from [RY, Lemma 4.1] and analytic perturbation theory $[\mathbf{K}]$.

Now let $\eta \in \mathcal{B}$ be a probability measure. Since $\Pi_{\lambda_{0}} \eta(1)=1$, we have $\Pi_{\lambda_{z}} \eta(1) \neq 0$ for $|z|$ sufficiently small [KL]. Thus, by (42),

$$
q(z)=\lim _{n \rightarrow \infty} \frac{1}{n} \log \eta\left(e^{z S_{n} g}\right)=\log \lambda_{z}
$$

proving the first statement of the theorem.

Since $q(z)$ is independent of $\eta$, we may write $q(z):=\lim _{n \rightarrow \infty}(1 / n) \log v_{0}\left(e^{z S_{n} g}\right)$. Now the computation of the derivatives of $q$ follows exactly as in the proof of [RY, Theorem 4.3].

Proof of Theorem 4. This follows directly from the Gartner-Ellis theorem [DZ] since $q(z)$ is a smooth function of $z$ for $z$ real and sufficiently small. 
6.2. Proof of Proposition 6.1. The estimates parallel those of $\S 4$. In order to avoid repetition, we indicate only where changes are necessary.

Weak norm. For $h \in \operatorname{Lip}^{u}(\Delta), E \in \mathcal{P}_{\ell, j}^{k}$ and $\varphi \in \mathcal{C}_{s}^{p}(E)$, if $n \leq \ell$, we follow (14),

$$
\begin{aligned}
\int_{E} \mathcal{L}_{g}^{n} h \varphi d m & =\int_{F^{-n} E} h e^{S_{n} g} \varphi \circ F^{n} d m \\
& \leq m\left(F^{-n} E\right)|h|_{w\left(\mathcal{P}_{\ell-n, j^{\prime}}^{k+n}\right)}\left|e^{S_{n} g}\right|_{\mathcal{C}_{s}^{p}\left(F^{-n} E\right)}\left|\varphi \circ F^{n}\right|_{\mathcal{C}_{s}^{p}\left(F^{-n} E\right)} .
\end{aligned}
$$

Since $\quad m(E)=m\left(F^{-n} E\right), \quad\left|\varphi \circ F^{n}\right|_{\mathcal{C}_{s}^{p}\left(F^{-n} E\right)} \leq|\varphi|_{\mathcal{C}_{s}^{p}(E)} \quad$ and $\quad\left|e^{S_{n} g}\right|_{\mathcal{C}_{s}^{p}\left(F^{-n} E\right)} \leq$ $C e^{n g_{0}} n|g|_{\mathcal{C}_{s}^{p}(\Delta)}$, we have

$$
\left|\mathcal{L}_{g}^{n} h\right|_{w\left(\mathcal{P}_{\ell, j}^{k}\right)} \leq C \beta_{0}^{n}|h|_{w\left(\mathcal{P}_{\ell-n, j^{\prime}}^{k+n}\right)} e^{n g_{0}}|g|_{\mathcal{C}_{s}^{p}(\Delta)}
$$

On the other hand, if $\ell=0$ then following (16) we estimate

$$
\begin{aligned}
\int_{E} \mathcal{L}_{g}^{n} h \varphi d m & =\sum_{E^{\prime} \subset F^{-n} E} \int_{E^{\prime}} h e^{S_{n} g} \varphi \circ F^{n} d m \\
& \leq \sum_{E^{\prime}} m\left(E^{\prime}\right) \beta_{0}^{-\ell^{\prime}}|h|_{w\left(\mathcal{P}_{\ell^{\prime}, j^{\prime}}^{k+n}\right)}\left|e^{S_{n} g}\right|_{\mathcal{C}_{s}^{p}\left(E^{\prime}\right)}\left|\varphi \circ F^{n}\right|_{\mathcal{C}_{s}^{p}\left(E^{\prime}\right)}
\end{aligned}
$$

where $E^{\prime} \in \mathcal{P}_{\ell^{\prime}, j^{\prime}}^{k+n}$ as in $§ 4.1$. Now (43) follows from (46) and (47) using (18).

Strong stable norm. Similarly following the estimates of $\S 4.2$, for $\varphi \in \mathcal{C}_{s}^{q}(E)$ we define $\bar{\varphi}$ as in (21). Then

$$
\int_{E} \mathcal{L}_{g}^{n} h \varphi d m=\sum_{E^{\prime} \subset F^{-n} E} \int_{E^{\prime}} h e^{S_{n} g}\left(\varphi \circ F^{n}-\bar{\varphi}\right) d m+\int_{E^{\prime}} h e^{S_{n} g} \bar{\varphi} d m .
$$

Now (44) follows from (20), (23) and (24).

Strong unstable norm. Taking $E_{r} \subset E_{k} \in \mathcal{P}_{\ell, j}^{k}$ and $\varphi \in \mathcal{C}_{s}^{p}\left(\Delta_{\ell, j}\right)$, we define $\tilde{\varphi}_{E_{r}}$ as in $\S 4.3$.

Case 1. $n \leq \ell$. Since $m\left(E_{r}\right)=m\left(F^{-n} E_{r}\right)$ and similarly for $E_{k}$, the estimate before (26) yields

$$
\begin{aligned}
& \left|f_{F^{-n} E_{r}} h e^{S_{n} g} \varphi \circ F^{n} d m-f_{F^{-n} E_{k}} h e^{S_{n} g}\left(\varphi \circ F^{n}\right)_{E_{r}^{n}} d m\right| \\
& \quad \leq\left\|h e^{S_{n} g}\right\|_{u\left(F^{-n} E_{k}\right)} \beta^{k+n-\ell}|\varphi|_{\mathcal{C}_{s}^{p}(E)} .
\end{aligned}
$$

Since $e^{S_{n} g} \in \operatorname{Lip}^{u}\left(F^{-n} E_{k}\right)$, by Lemma 3.3,

$$
\left\|h e^{S_{n} g}\right\|_{u\left(F^{-n} E_{k}\right)} \leq\|h\|_{S}\left(\operatorname{Lip}^{u}\left(\left.e^{S_{n} g}\right|_{F^{-n} E_{k}}\right)+H_{S}^{p}\left(e^{S_{n} g}\right)\right)+\|h\|_{u}\left|e^{S_{n} g}\right|_{\mathcal{C}_{S}^{p}(\Delta)} .
$$

Then since $\operatorname{Lip}^{u}\left(\left.e^{S_{n} g}\right|_{F^{-n} E_{k}}\right) \leq e^{n g_{0}} \operatorname{Lip}^{u}(g) /(1-\beta)$, we have

$$
\begin{aligned}
\left\|\mathcal{L}_{g}^{n} h\right\|_{u\left(\mathcal{P}_{\ell, j}^{k}\right)} \leq & \beta^{n}\|h\|_{u\left(\mathcal{P}_{\ell-n, j^{\prime}}^{k+n}\right)} C e^{n g_{0}}|g|_{\mathcal{C}_{s}^{p}(\Delta)} \\
& +\|h\|_{s}\left(C e^{n g_{0}}|g|_{\mathcal{C}_{s}^{q}(\Delta)}+e^{n g_{0}} \operatorname{Lip}^{u}(g) /(1-\beta)\right) .
\end{aligned}
$$


Case 2. $n>\ell$. Following $\$ 4.3$, we first consider the case $\ell=0$ and fix $E_{r} \subseteq E_{k} \subseteq \Delta_{0, j}$. We estimate the analogous terms of (27) one at a time.

The expression corresponding to (28) is $\leq C^{\prime} \beta^{n+k}\left\|h e^{S_{n} g}\right\|_{u\left(\mathcal{P}_{\ell^{\prime}, j^{\prime}}^{n+k}\right)}$; the term corresponding to (30) is $\leq C^{\prime}\left\|e^{S_{n} g} h\right\|_{s} \alpha^{k / 2}$; and the term corresponding to (33) is $\leq C\left\|e^{S_{n} g} h\right\|_{s} \alpha^{r(p-q) / 2 p}$. Putting these estimates together with (48) and using Lemma 3.3(ii) yields (45).

6.3. Proof of Lemma 6.2. Let $h \in \operatorname{Lip}^{u}(\Delta)$ and $E \in \mathcal{P}_{\ell, j}^{k}$ with $\ell \geq 1$. Notice that $F^{-1} E=E^{\prime} \in \mathcal{P}_{\ell-1, j^{\prime}}^{k+1}$. For any $\varphi \in \mathcal{C}_{S}^{p}(E)$, we have

$$
\begin{aligned}
\int_{E}\left(\mathcal{L}_{z}-\mathcal{L}_{0}\right) h \varphi d m & =\int_{E^{\prime}} h \varphi \circ F\left(e^{z g}-1\right) d m \\
& \leq m\left(E^{\prime}\right) \beta^{1-\ell}\|h\|_{s}|\varphi \circ F|_{\mathcal{C}_{s}^{q}\left(E^{\prime}\right)}\left|e^{z g}-1\right|_{\mathcal{C}_{s}^{q}\left(E^{\prime}\right)} .
\end{aligned}
$$

Since $F$ is rigid translation on $E^{\prime},|\varphi \circ F|_{\mathcal{C}_{s}^{q}\left(E^{\prime}\right)} \leq|\varphi|_{\mathcal{C}_{s}^{q}(E)}$ and $m\left(E^{\prime}\right)=m(E)$. It is straightforward to estimate $\left|e^{z g}-1\right|_{\mathcal{C}_{s}^{q}\left(E^{\prime}\right)} \leq e^{|z| g_{0}}|z||g|_{\mathcal{C}_{s}^{q}\left(E^{\prime}\right)}$. Taking the appropriate suprema,

$$
\left|\left(\mathcal{L}_{z}-\mathcal{L}_{0}\right) h\right|_{w\left(\mathcal{P}_{\ell, j}^{k}\right)} \leq\|h\|_{s} e^{|z| g_{0}}|z||g|_{\mathcal{C}_{s}^{q}(\Delta)} \quad \text { for } \ell \geq 1 .
$$

It remains to consider the case $\ell=0$. In this case, $F^{-1} E$ is the countable union of cylinders $E^{\prime} \in \mathcal{P}_{\ell^{\prime}, j^{\prime}}^{k+1}$. Let $\ell^{\prime}$ denote the level of $E^{\prime}$.

$$
\int_{E}\left(\mathcal{L}_{z}-\mathcal{L}_{0}\right) h \varphi d m \leq \sum_{E^{\prime} \subset F^{-1} E} m\left(E^{\prime}\right) \beta^{-\ell^{\prime}}\|h\|_{s}|\varphi \circ F|_{\mathcal{C}_{s}^{q}\left(E^{\prime}\right)}\left|e^{z g}-1\right|_{\mathcal{C}_{s}^{q}\left(E^{\prime}\right)}
$$

By (17), $|\varphi \circ F|_{\mathcal{C}_{s}^{q}\left(E^{\prime}\right)} \leq C_{0}|\varphi|_{\mathcal{C}_{s}^{q}(E)}$ and by (18), $m\left(E^{\prime}\right) \leq C m\left(E_{1}^{\prime}\right) m(E)$ where $E_{1}^{\prime}$ is the 1 -cylinder containing $E^{\prime}$. Thus

$$
\left|\left(\mathcal{L}_{z}-\mathcal{L}_{0}\right) h\right|_{w\left(\mathcal{P}_{0, j}^{k}\right)} \leq\|h\|_{s} C_{0} e^{|z| g_{0}}|z||g|_{\mathcal{C}_{s}^{q}(\Delta)} \sum_{\ell^{\prime}, j^{\prime}} \beta^{-\ell^{\prime}} m\left(\Delta_{\ell^{\prime}, j^{\prime}}\right) .
$$

This, together with (49), proves the lemma.

\section{Proof of Theorems 5 and 6}

Proof of Theorem 5. For any measure $\eta \in \mathcal{B}$, we may define its projection $\tilde{\eta}$ on $M$ by $\tilde{\eta}(A)=\eta\left(\pi^{-1} A\right)$ for all Borel $A \subset M$. In particular, if $\nu \in \mathcal{B}$ is an invariant measure for $F$, then $\pi_{*} \nu=\tilde{v}$ is an invariant measure for $f$ due to the relation $\pi \circ F=f \circ \pi$. Statement (i) now follows from Theorem 2(i), (ii).

That statement (ii) follows from Theorem 2(iv) is by now standard, although we follow a different route than the one used in [Y1] since we only require our test functions to be Hölder continuous along stable or unstable leaves separately. The crucial points are: (A) if $\psi \in C^{\zeta}\left(\Gamma^{u}(M)\right)$ and we choose $\beta_{0} \geq \alpha^{\zeta / 2}$, then its lift $\psi \circ \pi \in \operatorname{Lip}^{u}(\Delta)$; (B) if $\varphi \in C^{\zeta}\left(\Gamma^{s}(M)\right)$ and we choose $p \leq \zeta$, then $\varphi \circ \pi \in \mathcal{C}_{s}^{p}(\Delta)$.

To prove (A), take $x, y \in \gamma^{u} \in \Gamma^{u}\left(\Delta_{\ell, j}\right)$ and let $x_{0}=F^{-\ell} x, y_{0}=F^{-\ell} y$. Then

$$
|\psi(\pi x)-\psi(\pi y)| \leq H_{u}^{\zeta}(\psi) d(\pi x, \pi y)^{\zeta} \leq H_{u}^{\zeta}(\psi) d\left(f^{\ell}\left(\pi x_{0}\right), f^{\ell}\left(\pi y_{0}\right)\right)^{\zeta} .
$$


Now

$$
d\left(f^{\ell}\left(\pi x_{0}\right), f^{\ell}\left(\pi y_{0}\right)\right) \leq C_{0} \alpha^{s_{0}\left(\pi x_{0}, \pi y_{0}\right)-\ell} \leq C_{0} \alpha^{s(x, y)}
$$

by Property (P4)(a). So $\psi \circ \pi \in \operatorname{Lip}^{u}(\Delta)$ if $\beta_{0} \geq \alpha^{\zeta}$.

To prove (B), we take $x, y \in \gamma^{s} \in \Gamma^{s}\left(\Delta_{\ell, j}\right)$ and follow (50),

$$
|\varphi(\pi x)-\varphi(\pi y)| \leq H_{s}^{\zeta}(\varphi) d\left(f^{\ell}\left(\pi x_{0}\right), f^{\ell}\left(\pi y_{0}\right)\right)^{\zeta} \leq H_{s}^{\zeta}(\varphi) C_{0} \alpha^{\zeta \ell} d_{s}\left(x_{0}, y_{0}\right)^{\zeta}
$$

using property (P3). But $\alpha^{\ell} d_{s}\left(x_{0}, y_{0}\right)=d_{s}(x, y)$ so that $\varphi \circ \pi \in \mathcal{C}_{s}^{p}(\Delta)$ for all $p \leq \zeta$.

Statement (iii) follows from Theorem 1. Since $\xi \in \pi_{*} \mathcal{B}$ is a probability measure, we can find $\eta \in \mathcal{B}$ with $\Pi_{0} \eta(1)=1$ such that $\pi_{*} \eta=\xi$. Then $f_{*}^{n} \xi=f_{*}^{n}\left(\pi_{*} \eta\right)=\pi_{*}\left(F_{*}^{n} \eta\right)$ guarantees the required convergence.

To prove statement (iv), let $\eta \in \mathcal{B}$ be such that $\pi_{*} \eta=\xi$. For $\tilde{g} \in \mathcal{C}^{\zeta}(M)$, define its lift to $\Delta$ by $g=\tilde{g} \circ \pi$. Choosing $\beta_{0} \geq \alpha^{\zeta / 2}$ and $p=\zeta$ as in statement (ii) guarantees that $g \in \operatorname{Lip}^{u}(\Delta) \cap \mathcal{C}_{S}^{p}(\Delta)$. Since $e^{S_{n} \tilde{g}(\pi x)}=e^{S_{n} g(x)}$ for all $x \in \Delta$, Theorem 3 implies

$$
\lim _{n \rightarrow \infty} \frac{1}{n} \log \xi\left(e^{S_{n} \tilde{g}}\right)=\lim _{n \rightarrow \infty} \frac{1}{n} \log \eta\left(e^{S_{n} g}\right)=q(z) .
$$

Also,

$$
\left\{x \in \Delta:(1 / n) S_{n} g(x) \in[a, b]\right\}=\pi^{-1}\left\{x \in M:(1 / n) S_{n} \tilde{g}(x) \in[a, b]\right\}
$$

so, by Theorem 4 ,

$$
\lim _{n \rightarrow \infty} \frac{1}{n} \log \xi\left(\frac{1}{n} S_{n} \tilde{g}(x) \in[a, b]\right)=\lim _{n \rightarrow \infty} \frac{1}{n} \log \eta\left(\frac{1}{n} S_{n} g(x) \in[a, b]\right)=-\inf _{u \in[a, b]} I(u)
$$

for $[a, b] \subset\left[q^{\prime}\left(-\tau_{\max }\right), q^{\prime}\left(\tau_{\max }\right)\right]$ where $I(u)$ is the Legendre transform of $q(z)$.

Proof of Theorem 6. Let $\mu$ denote Lebesgue measure on $M$ and let $v$ denote the smooth invariant measure of the dispersing billiard map $f$. If $\xi=\psi v$ for some $\psi \in \mathcal{C}^{\zeta}\left(\Gamma^{u}(M)\right)$, then $\psi \circ \pi \in \operatorname{Lip}^{u}(\Delta)$ and so $\eta:=\psi \circ \pi \cdot \bar{v} \in \mathcal{G}$ by Lemma 5.2. Since $\pi_{*} \eta=\xi$, we have $\xi \in \pi_{*} \mathcal{B}$ so that the convergence result and large-deviation estimates follow from Theorem 5. It remains to prove the theorem for measures of the form $\xi=\psi \mu$ for $\psi \in \mathcal{C}^{\zeta}\left(\Gamma^{u}(M)\right)$. This will follow immediately once we prove the theorem for $\mu$.

Recall that the canonical coordinates used in dispersing billiards are $(r, \phi)$ where $r$ indicates position along the boundary of a scatterer (oriented clockwise) and $\phi$ is the angle an outgoing trajectory makes with the unit normal on the boundary. In these coordinates, $\mu=(k / \cos \phi) v$ where $k$ is a normalizing constant. Since $\bar{\psi}=k / \cos \phi$ is not bounded, $\mu \notin \pi_{*} \mathcal{G}$; however, by approximating $\mu$ by measures in $\pi_{*} \mathcal{G}$, we can obtain the stated results.

Proof of statement (i). Let $N_{\varepsilon}$ be an $\varepsilon$-neighborhood of $\phi= \pm \pi / 2$ in the phase space of the billiard map. Note that $\mu\left(N_{\varepsilon}\right)=\mathcal{O}(\varepsilon)$. Let

$$
\psi_{\varepsilon}=\min \left\{\bar{\psi}, \frac{k}{\cos (\pi / 2-\varepsilon)}\right\}
$$

and note that $\psi_{\varepsilon}$ is Lipschitz on $M$. Let $\mu_{\varepsilon}=\psi_{\varepsilon} \nu$. Then since $\mu_{\varepsilon} \in \pi_{*} \mathcal{G}$, for $\varphi \in C^{0}(M)$,

$$
\lim _{n \rightarrow \infty} f_{*}^{n} \mu(\varphi)=\lim _{n \rightarrow \infty} f_{*}^{n} \mu_{\varepsilon}(\varphi)+\left(\mu-\mu_{\varepsilon}\right)\left(\varphi \circ f^{n}\right)=v(\varphi)+|\varphi|_{\infty} \mathcal{O}(\varepsilon)
$$

by Theorem 5(iii). Sending $\varepsilon \rightarrow 0$ proves statement (i). 
Proof of statement (ii). Let $A_{n}(a, b)=\left\{x \in M:(1 / n) S_{n} g(x) \in[a, b]\right\}$. Then
$\lim _{n \rightarrow \infty} \mu\left(A_{n}(a, b)\right)=\lim _{n \rightarrow \infty} \mu_{\varepsilon}\left(A_{n}(a, b)\right)+\left(\mu-\mu_{\varepsilon}\right)\left(A_{n}(a, b)\right)=\inf _{u \in[a, b]}-I(u)+\mathcal{O}(\varepsilon)$,

by Theorem 5(iv). The large-deviation result follows by letting $\varepsilon \rightarrow 0$.

Acknowledgements. The author would like to thank L.-S. Young for helpful discussions, C. Liverani for introducing him to the functional approach, and MSRI, Berkeley, where this work was begun. This research is partially supported by NSF grant DMS-0801139.

\section{REFERENCES}

[B] V. Baladi. Positive Transfer Operators and Decay of Correlations (Advanced Series in Nonlinear Dynamics, 16). World Scientific, Singapore, 2000.

[BT] V. Baladi and M. Tsujii. Anisotropic Hölder and Sobolev spaces for hyperbolic diffeomorphisms. Ann. Inst. Fourier (Grenoble) 57 (2007), 127-154.

[BY] M. Benedicks and L.-S. Young. Markov extensions and decay of correlations for certain Hénon maps. Astérique 261 (2000), 13-56.

[BKL] M. Blank, G. Keller and C. Liverani. Ruelle-Perron-Frobenius spectrum for Anosov maps. Nonlinearity 15(6) (2001), 1905-1973.

[BDM] H. Bruin, M. Demers and I. Melbourne. Existence and convergence properties of physical measures for certain dynamical systems with holes. Ergod. Th. \& Dynam. Sys. to appear.

[C1] N. Chernov. Statistical properties of piecewise smooth hyperbolic systems in high dimensions. Discrete Contin. Dyn. Syst. 5 (1999), 425-448.

[C2] N. Chernov. Sinai billiards under small external forces. Ann. Henri Poincaré 2(2) (2001), 197-236.

[C3] N. Chernov. Advanced statistical properties of dispersing billiards. J. Stat. Phys. 122 (2006), 1061-1094.

[CY] N. Chernov and L.-S. Young. Decay of correlations for Lorenz gases and hard balls. Hard Ball Systems and the Lorenz Gas (Encyclopaedia of Mathematical Sciences, 101). Ed. D. Szasz. Springer, Berlin, 2000, pp. 89-120.

[DZ] A. Dembo and O. Zeitouni. Large Deviations Techniques and Applications, 2nd edn (Applications of Mathematics, 38). Springer, New York, 1998.

[DL] M. Demers and C. Liverani. Stability of statistical properties for two-dimensional piecewise hyperbolic maps. Trans. Amer. Math. Soc. 360(9) (2008), 4777-4814.

[DWY] M. Demers, P. Wright and L.-S. Young. Escape rates and physically relevant measures for billiards with small holes. Comm. Math. Phys. to appear.

[DF] W. Doeblin and R. Fortet. Sur des chaînes à liaisons complète. Bull. Soc. Math. France 65 (1937), 132-148.

[GL] S. Gouëzel and C. Liverani. Banach spaces adapted to Anosov systems. Ergod. Th. \& Dynam. Sys. 26(1) (2006), 189-217.

[HH] H. Hennion and L. Hervé. Limit Theorems for Markov Chains and Stochastic Properties of Dynamical Systems via Quasi-Compactness (Lecture Notes in Mathematics, 1766). Springer, Berlin, 2001.

[HM] M. Holland and I. Melbourne. Central limit theorems and invariance principles for Lorenz attractors. J. Lond. Math. Soc. (2) 76 (2007), 345-364.

[IM] C. T. Ionescu-Tulcea and G. Marinescu. Théorème ergodique pour des classes d'opérations non complètement continues. Ann. of Math. (2) 52 (1950), 140-147.

[K] T. Kato. Perturbation Theory for Linear Operators, 2nd edn (Grundlehren der mathematischen Wissenchaften, 132). Springer, Berlin, 1984.

[K] G. Keller. On the rate of convergence to equilibrium in one-dimensional systems. Comm. Math. Phys. 96(2) (1984), 181-193.

[KL] G. Keller and C. Liverani. Stability of the spectrum for transfer operators. Ann. Sc. Norm. Super. Pisa Cl. Sci. (4) 28 (1999), 141-152. 
[KN] G. Keller and T. Nowicki. Spectral theory, zeta functions and the distribution of periodic points for Collet-Eckmann maps. Comm. Math. Phys. 149 (1992), 31-69.

[LY] A. Lasota and J. A. Yorke. On the existence of invariant measures for piecewise monotonic transformations. Trans. Amer. Math. Soc. 186 (1963), 481-488.

[MN] I. Melbourne and M. Nicol. Large deviations for nonuniformly hyperbolic systems. Trans. Amer. Math. Soc. 360 (2008), 6661-6676.

[OP] S. Orey and S. Pelikan. Deviations of trajectory averages and the defect in Pesin's formula for Anosov diffeomorphisms. Trans. Amer. Math. Soc. 315(2) (1989), 741-753.

[N] S. V. Nagaev. Some limit theorems for stationary Markov chains. Theory Probab. Appl. 11(4) (1957), 378-406.

[RY] L. Rey-Bellet and L.-S. Young. Large deviations in nonuniformly hyperbolic dynamical systems. Ergod. Th. \& Dynam. Sys. 28 (2008), 587-612.

[R] H. H. Rugh. The correlation spectrum for hyperbolic analytic maps. Nonlinearity 5(6) (1992), 1237-1263.

[W] P. Walters. Ergodic Theory: Introductory Lectures (Lecture Notes in Mathematics, 458). Springer, Berlin, 1975.

[WY] Q. Wang and L.-S. Young. Nonuniformly expanding 1D maps. Comm. Math. Phys. 264 (2006), 255-282.

[Y1] L.-S. Young. Statistical properties of dynamical systems with some hyperbolicity. Ann. of Math. (2) 147 (1998), 585-650.

[Y2] L.-S. Young. Recurrence times and rates of mixing. Israel J. Math. 110 (1999), 153-188. 